This article was published in an Elsevier journal. The attached copy

is furnished to the author for non-commercial research and education use, including for instruction at the author's institution, sharing with colleagues and providing to institution administration.

Other uses, including reproduction and distribution, or selling or licensing copies, or posting to personal, institutional or third party websites are prohibited.

In most cases authors are permitted to post their version of the article (e.g. in Word or Tex form) to their personal website or institutional repository. Authors requiring further information regarding Elsevier's archiving and manuscript policies are encouraged to visit: 


\title{
Static analysis of beam structures under nonlinear geometric and constitutive behavior
}

\author{
P. Mata, S. Oller *, A.H. Barbat \\ Technical University of Catalonia, UPC, Edificio C1, Campus Nord, Gran Capitá s/n, 08034 Barcelona, Spain
}

Received 6 July 2006; received in revised form 4 May 2007; accepted 10 May 2007

Available online 23 May 2007

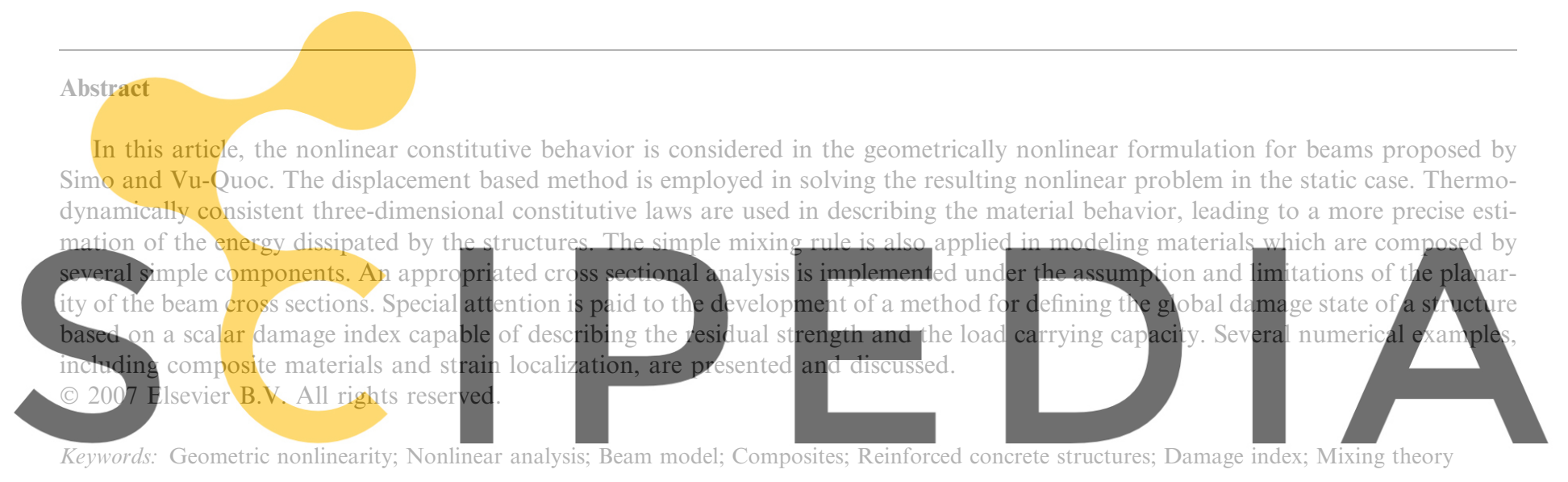

Register for free at https//www.scipedia.com to download the version without the watermark

\section{Introduction}

The three-dimensional nonlinear analysis of beam structures has captured the interest of many researchers during the past decades. As mentioned by Nukala et al. [50], many contributions have been focused on the formulation of geometrically consistent models of beams undergoing large displacements and rotations, but considering that the material behavior remains elastic and, therefore, employing simplified linear constitutive relations in terms of cross sectional forces and moments. On the contrary, the constitutive nonlinearity in beams has been described by means of concentrated and distributed models, both of them formulated, in the most cases, for small strain and small displacement kinematic hypothesis. Only a few works have been carried out using fully geometrical and material nonlinear formulations for beams, but they have been mainly

\footnotetext{
* Corresponding author. Tel.: +34 934017401; fax: +34 934011048. E-mail address: sergio.oller@upc.edu (S. Oller).
}

focused on plasticity. In the following we briefly describe the most frequently used formulations for simulating both geometric and constitutive nonlinear mechanical behavior of beam structures, addressing the most relevant contributions and highlighting their limitations.

One of the most invoked geometrically exact formulations is that of Simo [58], which generalize to the three-dimensional dynamic case the formulation originally developed by Reissner [51,52] for the plane static problem. According to Simo, this formulation constitutes a specific parametrization of the three-dimensional case of the classical Kirchhoff-Love rod model due to Antman [1], employing a director type approach for describing the configuration of the beam cross sections during the motion, which allows to consider finite shearing and finite extension. Posteriorly, Simo and Vu-Quoc $[59,60]$ implemented the numerical integration of the equations of motion of the rods in the context of the finite element (FE) framework for the static and dynamic cases. They considered a straight and unstressed rod as reference configuration and the hypothesis of planar sections, neglecting any kind of warping. The resulting 
geometric stiffness, although non-symmetric away from equilibrium [61], is always symmetric in the equilibrium configuration.

A great amount of works on both the theory and numerical implementation of the geometrically exact formulations for beams have been developed starting from the Reissner and Simo and Vu-Quoc works. Particularly, interesting developments have been carried out by Ibrahimbegović to extend the formulation given in Ref. [58] to the case of a curved reference configuration of the rod [24] and proposing alternative numerical treatments for the parametrization of the rotations [26]. Jelenić and Saje [30] develop a formulation based on the so called generalized principle of virtual work, eliminating the displacement variables of the model and retaining only rotational degrees of freedom, avoiding thus the shear locking phenomenon in the numerical simulations.

The usual discretization procedures applied in implementing the strain measures in the FE method violates the objectivity condition of this tensor [53]; Jelenic and Crisfield in Refs. $[14,29]$ propose a remedy for this problem. In [27] several improvements in finite element implementations are addressed to ensure the invariance of the continuum problem. In [7] a new objective beam finite ele-

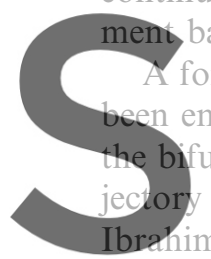
formulation equivalent to that
employed by Cardona and $\mathbf{1}$
of flexible mechanisms with
imbegovic et al. in $[25,28]$ fo
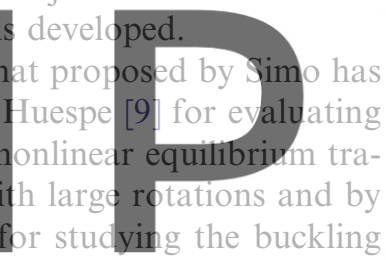

and post buckling behavior of framed structures. A comRegister for fre bat heen tangent and sccant formulations

out by Pérez Morán [40] along with a generalization of the original formulations allowing to use any kind of suitable parametrization for the rotational field. It is interesting to note that other kind of geometrically nonlinear formulations for beams, the so called inexact or co-rotational are discussed in detail by Crisfield in Ref. [13].

Works on constitutive nonlinearity have progressed based on a different approach, that is, lumped and distributed plasticity models [48]. The lumped plasticity models consider linear elastic structural elements equipped with plastic hinges at the ends. Some of these models have been extended to consider a wide variety of failure criteria; an example is shown in the work of Hyo-Gyoung Kwank and Sun-Pil Kim [22] where a moment-curvature relationship for the study of reinforced concrete (RC) beams subjected to cyclic loading is defined. This method is recommended by certain authors due to its numerical efficiency when compared with the full three-dimensional (3D) formulation of the problem. But it is important to note that the nonlinear constitutive laws are valid only for specific geometries of the beam cross sections and that usually, the thermodynamical basis of the material behavior are violated [21].

In the case of distributed plasticity models, the constitutive nonlinearity is evaluated at a fixed number of cross sec- tions along the beam axis, allowing to obtain a distributed nonlinear behavior along the structural elements. These sections are divided into a number of control points corresponding to fibers directed along the beam's axis. Therefore, this approach is frequently referred as fiber approach [56]. The employment of fibers allows predicting a more realistic strain-stress state at the cross sectional level, but it requires the definition of uniaxial constitutive laws for each material point. A combination of both models, applied to the study of the collapse loads of RC structures, is proposed by Kim and Lee [33].

Two versions of the distributed plasticity models can be found in literature: the stiffness (displacement based) and flexibility (force-based) methods [48]. The first one is based on the interpolation of the strain field along the elements. A precise representation of forces and moments requires a refined FE mesh for each structural element in which inelastic behavior is expected to appear. In the flexibility method, the sectional forces and moments are obtained interpolating the nodal values and satisfying the equilibrium equations even in the constitutive nonlinear range [55].

Both approaches are affected by the strain localization phenomenon when materials with softening behavior are employed. In the stiffness method, localization occurs in a specific elentent and, in the case of the flexibility method,
nonlinearity is concentrated in the volume associated to a
specific section of the element undergoing softening. In
any case, the whole structural response becomes mesh
dependent if no appropriate corrections are considered.
Several techniques have been proposed for ensuring objectivity of the structural element response: Scott and Fenves downdevelgp new integration metho based on the Gauss based elements; Hanganu et al. [21] and Barbat et al. [6] regularize the energy dissipated at material point level, limiting its value to the specific fracture energy of the material [42]. These methods ensure that the whole structural response remains objective, but the length of the zone where softening occurs is still mesh dependent. Recently, some developments employing strong discontinuities have been applied to the study of beam models but considering constitutive laws in terms of sectional forces, as it can be seen in [2] and in the references given therein.

One of the most common limitations of the distributed formulations lies in the fact that inelasticity is defined for the component of the strain acting in the direction normal to the face of the cross section and, therefore, the shearing components of the stress are treated elastically. This assumption does not allows to simulate the nonlinear coupling between different stress components at constitutive level, resulting in models where sectional shear forces and torsion moments are transmitted elastically across the elements $[15,41]$. Moreover, it predefines the way in which the failure of the members occurs, limiting the participation of shear forces to the equilibrium. A comparative study of different plasticity models applied to earthquake analysis of buildings can be reviewed in [16]. 
Works considering both constitutive and geometric nonlinearity are scarce and they have been mainly restricted to plasticity $[47,54]$. For example, in $[8,17]$ a higher order approximation is used for the calculation of the axial strain in truss elements and uniaxial constitutive descriptions are used for the materials. Outstanding works considering warping of arbitrary sections made of rate dependent and rate independent elastic-plastic material are proposed by Simo et al. [63] and Gruttmann et al. [20], respectively, while Kumar and White [50] develop a mixed finite element for studying the stability of steel structures.

In this work, the general nonlinear constitutive behavior is included in the static version of the geometrically exact formulation for beams proposed by Simo [58], considering an intermediate curved reference configuration between the straight reference beam and the current configuration. The displacement based method is used for solving the resulting nonlinear problem. Plane cross sections remain plane after the deformation of the structure; therefore, no cross sectional warping is considered, avoiding to include additional warping variables in the formulation or iterative procedures to obtain corrected cross sectional strain fields. An appropriated cross sectional analysis is applied for obtaining the cross sectional forces and moments [6] and the consistent tangential tensors in the linearized problem.
Thernodynamically consistent constitutive laws are used
in describing the material behavior, which dllows to obtain
a more rational estimation of the energy dissipated by the
structures. The simple mixing rule is also considered in
modeling materials which are composed by several simple components. Special attention is paid to the obtention of

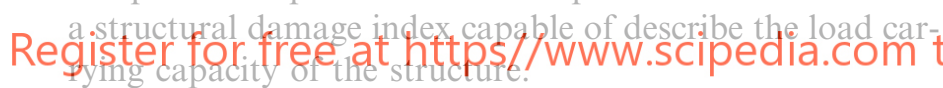

2. Finite strain formulation for initially curved beams

The formulation of Simo and Vu-Quoc [58,59] for beams that can undergo large deformations in space is expanded considering an intermediate (free of stress) curved reference configuration between the straight reference beam and the deformed beam in the current configuration [24]. The description of both the geometry and the kinematics of the beam is developed in the nonlinear differential manifold $\mathbb{R}^{3} \times S O(3)$, where the rotation manifold is denoted by $S O(3)$ [59]. Details about the theory of finite rotations can be reviewed in $[5,38,58]$.

Let $\left\{\widehat{E}_{i}\right\}$ and $\left\{\hat{e}_{i}\right\}(i=1 \ldots 3)$ be the spatially fixed (inertial) material and spatial frames, respectively. The straight reference beam is defined simply by the curve $\hat{\varphi}_{00}=S \widehat{E}_{1}$, with $S \in[0, L] \subset \mathbb{R}$ its arch-length coordinate. Beam cross sections are described by means of the coordinates $\xi_{\beta}$ directed along $\left\{\widehat{E}_{\beta}\right\}(\beta=2,3)$ and, therefore, the position vector $\widehat{X}$ of any material point is $\widehat{X}=S \widehat{E}_{1}+$ $\sum_{\beta=2}^{3} \xi_{\beta} \widehat{E}_{\beta}$.

The curved reference beam is defined by means of a spatially fixed curve with position vector given by $\hat{\varphi}_{0}=\sum_{i=1}^{3} \varphi_{0 i}(S) \hat{e}_{i} \in \mathbb{R}^{3}$, where $S$ and $[0, L]$ are the same as for the straight reference beam. Additionally, each point on this curve has rigidly attached an orthogonal local frame $\hat{t}_{0 i}(S)=\Lambda_{0} \widehat{E}_{i} \in \mathbb{R}^{3}$, where $\Lambda_{0} \in S O(3)$ is the orientation tensor. The beam cross section $\mathscr{A}(S)$ is defined considering the local coordinate system $\xi_{\beta}$ but directed along $\left\{\hat{t}_{0 \beta}\right\}$. The planes of the cross sections are normal to the vector tangent to the reference curve, i.e., $\hat{\varphi}_{0,},_{S}=\hat{t}_{01}(S)$, $\forall S \in[0, L]$, (where the symbol $(\bullet),{ }_{x}$ is used to denote partial differentiation of $(\bullet)$ with respect to the variable $x$ ). The position vector $\hat{x}_{0}$ of any material point on the curved reference beam is

$\hat{x}_{0}=\hat{\varphi}_{0}+\sum_{\beta=2}^{3} \boldsymbol{\Lambda}_{0} \xi_{\beta} \widehat{E}_{\beta} \in \mathscr{A}(S) \times[0, L]$

The motion deforms the centroid line of the curved reference beam from $\hat{\varphi}_{0}(S)$ to $\hat{\varphi}(S, t)$ (at time $t$ ) adding a translational displacement $\hat{u}(S) \in \mathbb{R}^{3}$, i.e. $\hat{\varphi}(S, t)=\hat{\varphi}_{0}(S)+$ $\hat{u}(S, t)$. The local orientation frame is simultaneously rotated together with the beam cross section, from $\Lambda_{0}(S)$ to $\boldsymbol{\Lambda}(S, t)$ by means of the incremental rotation tensor $\boldsymbol{\Lambda}_{n}(S, t)[3,4]$, i.e., $\boldsymbol{\Lambda}=\boldsymbol{\Lambda}_{n} \boldsymbol{\Lambda}_{0} \equiv \sum_{i=1}^{3} \hat{t}_{i} \otimes \widehat{E}_{i} \in S O(3)$ for the spatial updating of the new compound rotation (see Fig. 1).

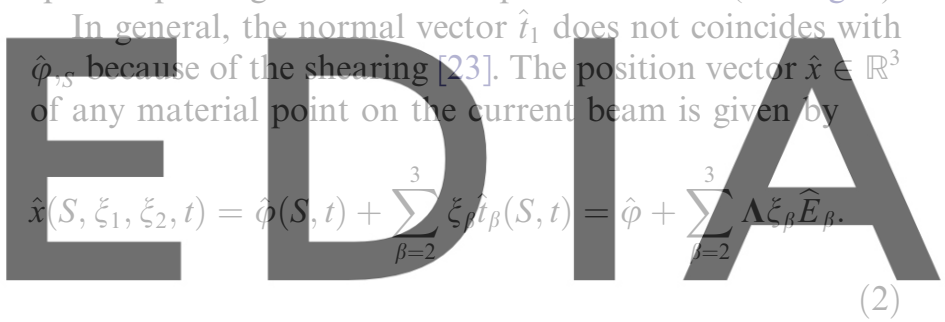

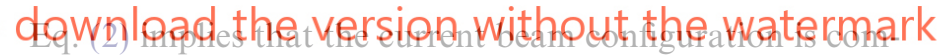
pletely determined by the pairs $(\hat{\varphi}, \Lambda) \in \mathbb{R}^{3} \times S O(3)$ $[10,38,59]$ and the spatial placement of the beam is defined as

$$
\mathscr{B}_{\mathrm{t}}:=\left\{\hat{x}(S, t) \in \mathbb{R}^{3} \mid \hat{\varphi}_{0}(S, t)+\sum_{\beta=2}^{3} \boldsymbol{\Lambda}(S, t) \xi_{\beta} \widehat{E}_{\beta} ;\left(S, \xi_{2}, \xi_{3}\right) \in[0, L] \times \mathscr{A}\right\} .
$$

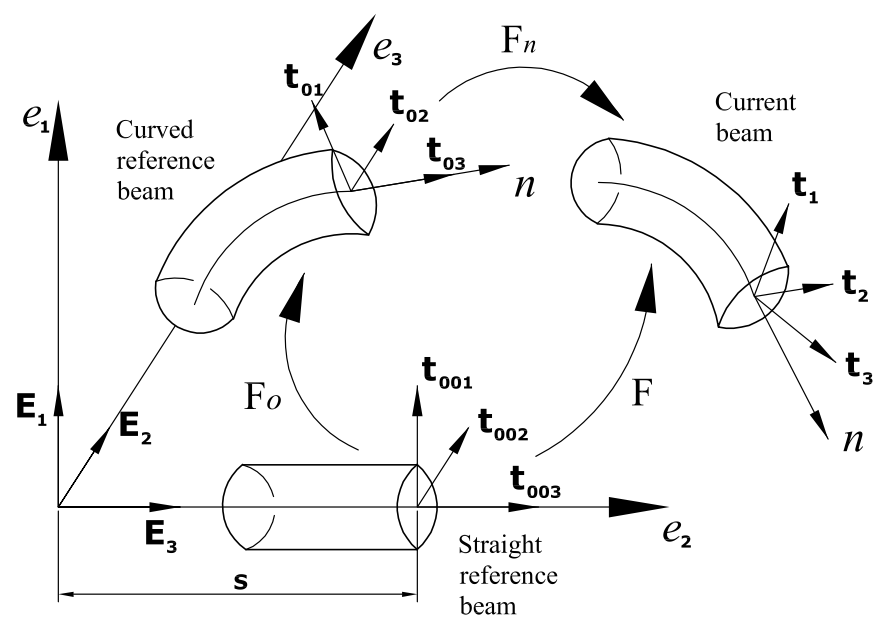

Fig. 1. Configurational description of the beam. 
Table 1

Spatial and material descriptions of curvatures

\begin{tabular}{ll}
\hline Material & Spatial \\
\hline$\widetilde{\boldsymbol{\Omega}}_{0} \equiv \boldsymbol{\Lambda}_{0}^{\mathrm{T}} \boldsymbol{\Lambda}_{0, S}=\boldsymbol{\Lambda}_{0}^{\mathrm{T}} \widetilde{\boldsymbol{\omega}}_{0} \boldsymbol{\Lambda}_{0}$ & $\widetilde{\boldsymbol{\omega}}_{0} \equiv \boldsymbol{\Lambda}_{0, S} \boldsymbol{\Lambda}_{0}^{\mathrm{T}}$ \\
$\widetilde{\boldsymbol{\Omega}}_{n} \equiv \boldsymbol{\Lambda}_{n}^{\mathrm{T}} \boldsymbol{\Lambda}_{n}, \boldsymbol{\Lambda}^{\mathrm{T}} \widetilde{\boldsymbol{\omega}}_{n} \boldsymbol{\Lambda}=\boldsymbol{\Lambda}^{\mathrm{T}} \boldsymbol{\Lambda},{ }_{S}-\boldsymbol{\Lambda}_{0}^{\mathrm{T}} \boldsymbol{\Lambda}_{0}, S$ & $\widetilde{\boldsymbol{\omega}}_{n} \equiv \boldsymbol{\Lambda}_{n}, \boldsymbol{\Lambda}_{n}^{\mathrm{T}}$ \\
$\widetilde{\boldsymbol{\Omega}} \equiv \boldsymbol{\Lambda}^{\mathrm{T}} \boldsymbol{\Lambda}, \boldsymbol{\Lambda}_{S} \widetilde{\boldsymbol{\omega}} \boldsymbol{\Lambda}=\widetilde{\boldsymbol{\Omega}}_{n}+\widetilde{\boldsymbol{\Omega}}_{0}$ & $\widetilde{\boldsymbol{\omega}} \equiv \boldsymbol{\Lambda},{ }_{S} \boldsymbol{\Lambda}^{\mathrm{T}}=\widetilde{\boldsymbol{\omega}}_{n}+\boldsymbol{\Lambda}_{n} \widetilde{\boldsymbol{\omega}}_{0} \boldsymbol{\Lambda}_{n}^{\mathrm{T}}$
\end{tabular}

The tangent space to $\mathscr{B}_{\mathrm{t}}$ at $\hat{x}$ is given by $T_{\hat{x}} \mathscr{B}_{\mathrm{t}}:=\left\{\delta \hat{x} \in \mathbb{R}^{3} \mid \hat{x} \in \mathscr{B}_{\mathrm{t}}\right\}$ for any variation $\delta \hat{x}$ obtained from the kinematically admissible variation of the variables defining current configuration i.e. $(\delta \hat{\varphi}, \delta \hat{\theta}) \in \mathbb{R}^{3} \times T_{\Lambda}^{\mathrm{spa}}$, so that $\delta \boldsymbol{\Lambda}=(\delta \hat{\theta} \times \boldsymbol{\Lambda}) \in T_{\boldsymbol{\Lambda}}^{\mathrm{spa}} S O(3) \quad[38] .{ }^{1}$ Fixing the time $t=t_{0}$ or $t=t_{00}$, it is possible to define the initial placement $\mathscr{B}_{0}$ and its tangent space $T \widehat{\widehat{X}}_{X} \mathscr{B}_{0}$ for the curved reference beam and $\mathscr{B}_{00}$ and $T \widehat{C}_{00}$ for the straight reference beam. Table 1 summarizes the curvature tensors representing the orientation change rate of the cross section with respect to the coordinate $S$, obtained from $\Lambda=\Lambda_{n} \Lambda_{0}$ by deriving with respect to $S$ and considering the pull-back and pushforward relationships between the material and spatial descriptions $[38,59]$. In this table, $\boldsymbol{\Omega}, \boldsymbol{\Omega}_{0}, \boldsymbol{\Omega}_{n} \in$ so(3), with so(3) being the linear space of all skew-symmetric tensors,

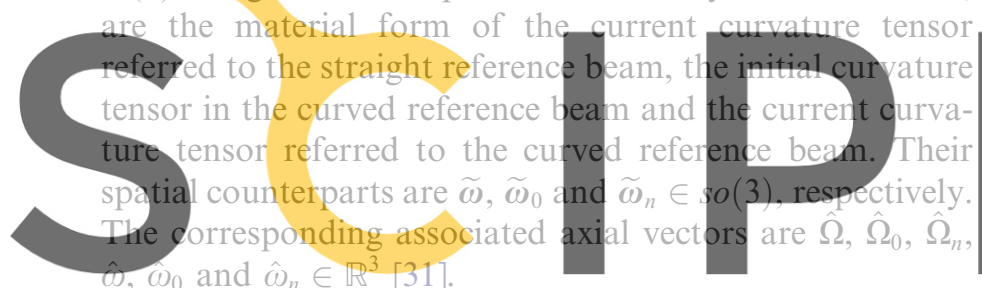

By the other hand, the deformation gradient (tensor) is

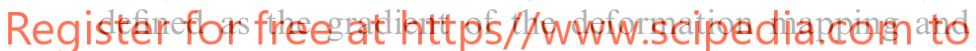
determines the strain measures at any material point of the beam cross section [59]. The deformation gradients of the curved reference beam and of the current beam referred to the straight reference configuration are denoted by $\mathbf{F}_{0}$ and F, respectively. Explicit expressions for them are [31]

$$
\begin{aligned}
\mathbf{F}_{0}= & {\left[\hat{\varphi}_{0}, \hat{t}_{01}+\sum_{\beta=2}^{3} \widetilde{\boldsymbol{\omega}}_{0} \xi_{\beta} \hat{t}_{0 \beta}\right] \otimes \widehat{E}_{1} } \\
& +\boldsymbol{\Lambda}_{0} \in T_{\widehat{X}} \mathscr{B}_{0} \otimes T_{\widehat{X}} \mathscr{B}_{00}, \\
\mathbf{F}= & {\left[\hat{\varphi},_{S}-\hat{t}_{1}+\sum_{\beta=2}^{3} \widetilde{\boldsymbol{\omega}} \xi_{\beta} \hat{t}_{\beta}\right] \otimes \widehat{E}_{1}+\boldsymbol{\Lambda} \in T_{\hat{x}} \mathscr{B} \otimes T_{\widehat{X}} \mathscr{B}_{00} . }
\end{aligned}
$$

It is worth noting that in Eq. (4b) the term defined as $\hat{\gamma}=\hat{\varphi},_{S}-\hat{t}_{1}$ corresponds to the reduced strain measure of shearing and elongation $[31,58]$ which, in conjunction with the curvature tensor $\widetilde{\boldsymbol{\omega}}$, allows measuring the strain state $\hat{\varepsilon}$ existing in each material point of the current beam cross section referred to the straight reference beam, i.e.

\footnotetext{
${ }^{1}$ The symbol $T_{\Lambda}^{\text {spa }} S O(3)$ denotes the spatial form of the tangential space to $\mathrm{SO}(3)$, with base point $\Lambda$, and $T_{\Lambda}^{\mathrm{spa}}$ is the spatial linear vector space of rotations, with base point $\boldsymbol{\Lambda}$, as described in Ref. [38].
}

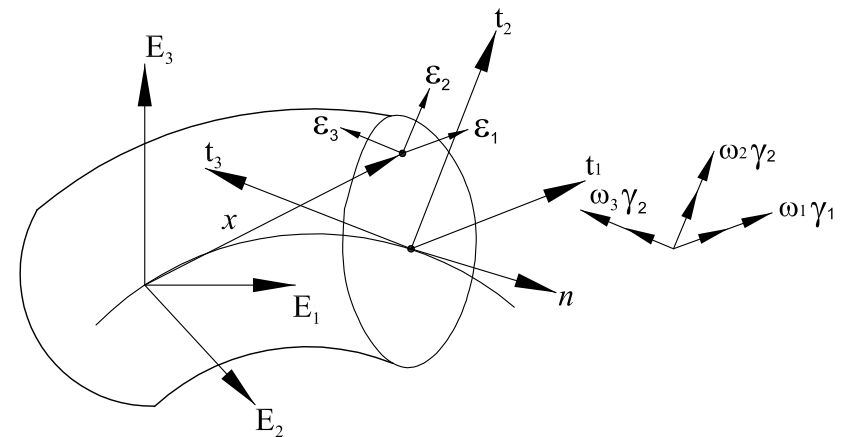

Fig. 2. Spatial form of the reduced strain measures $\hat{\gamma}$ and $\hat{\omega}$.

$\hat{\varepsilon}=\hat{\gamma}+\sum_{\beta=2}^{3} \widetilde{\boldsymbol{\omega}} \xi_{\beta} \hat{t}_{\beta}$ (see Fig. 2). The material description ${ }^{2}$ of $\mathbf{F}_{0}, \mathbf{F}, \hat{\gamma}$ and $\hat{\varepsilon}$ can be obtained by means of the pull-back operation as: $\quad \mathbf{F}^{\mathrm{m}}=\boldsymbol{\Lambda}_{0}^{\mathrm{T}} \mathbf{F}_{0}, \quad \mathbf{F}^{\mathrm{m}}=\boldsymbol{\Lambda}^{\mathrm{T}} \mathbf{F}, \quad \hat{\Gamma}=\boldsymbol{\Lambda}^{\mathrm{T}} \hat{\gamma} \quad$ and $\hat{\mathscr{E}}=\Lambda^{\mathrm{T}} \hat{\varepsilon}$, respectively

The deformation gradient $\mathbf{F}_{n}:=\mathbf{F F}_{0}^{-1}: T_{\widehat{X}} \mathscr{B}_{0} \rightarrow T_{\hat{x}} \mathscr{B}_{\mathrm{t}}$ relating the differential arch-length elements of the curved reference configuration with the current placement is responsible for the development of strains, and it can be expressed as $[31,43,62]$

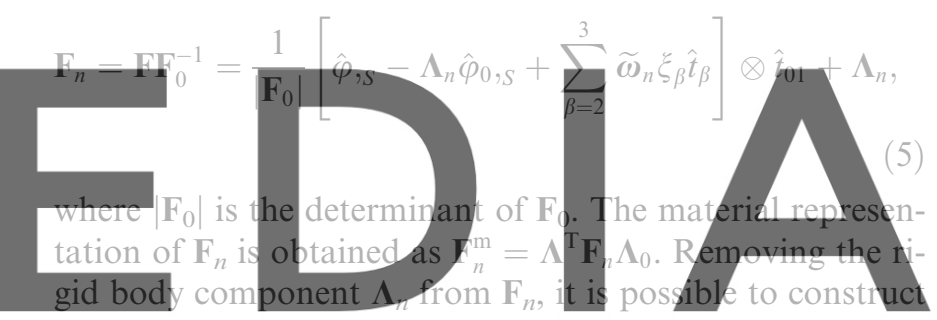

the strain tensor $\varepsilon_{n}=\mathbb{F}_{n}-\Lambda_{n} \in T_{\hat{x}} \mathscr{B}_{\mathrm{t}} \otimes T_{\widehat{X}} \mathscr{B}_{0}$, conjugated

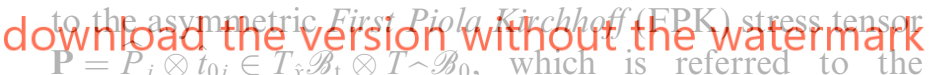
curved reference beam [58]. $\widehat{P}_{j}$ is the corresponding FPK stress vector acting on the deformed face in the current beam corresponding to the normal $\hat{t}_{0 j}$ in the curved reference configuration.

By other hand, the spatial strain vector acting on the current beam cross section relative to an element of area in the curved reference beam is obtained as $\hat{\varepsilon}_{n}=\boldsymbol{\varepsilon}_{n} \hat{t}_{01}$. The spatial form of the stress resultant $\hat{n}$ and the stress couple $\hat{m}$ vectors can be estimated from the stress vector $\widehat{P}_{1}$ according to

$\hat{n}(S)=\int_{\mathscr{A}} \widehat{P}_{1} \mathrm{~d} \mathscr{A} ; \quad \hat{m}(S)=\int_{\mathscr{A}}(\hat{x}-\hat{\varphi}) \times \widehat{P}_{1} \mathrm{~d} \mathscr{A}$.

The material form of $\widehat{P}_{j}, \hat{\varepsilon}_{n}, \hat{n}$ and $\hat{m}$ are obtained by means of the pull-back operation as $\widehat{\mathscr{E}}_{n}=\Lambda^{\mathrm{T}} \widehat{\varepsilon}_{n}, \widehat{P}_{j}^{\mathrm{m}}=\Lambda^{\mathrm{T}} \widehat{P}_{j}$, $\hat{m}^{\mathrm{m}}=\boldsymbol{\Lambda}^{\mathrm{T}} \hat{n}$ and $\hat{m}^{\mathrm{m}}=\boldsymbol{\Lambda}^{\mathrm{T}} \hat{m}$, respectively.

According to the developments presented in Ref. [1], the classical form of the equations of motion of the Cosserat beams for the static case are

\footnotetext{
${ }^{2}$ It is worth to note that even when the deformation gradient is intrinsically two point tensors, mathematically it is possible to obtain its material counterpart by means of pulling back by $\boldsymbol{\Lambda}$ its spatial leg to the material configuration $[31,38]$.
} 
$\hat{n},_{S}+\hat{n}_{\mathrm{p}}=0, \quad \hat{m},_{S}+\hat{\varphi},_{S} \times \hat{n}+\hat{m}_{\mathrm{p}}=0$,

where $\hat{n}_{\mathrm{p}}(S, t)$ and $\hat{m}_{\mathrm{p}}(S, t)$ are the external body force and body moment per unit of reference length at time $t$.

Considering a kinematically admissible variation $h \equiv(\delta \hat{\varphi}, \delta \hat{\theta}) \in \mathbb{R}^{3} \times T_{\Lambda}^{\mathrm{spa}}$ of the pair $(\hat{\varphi}, \boldsymbol{\Lambda})[59]$, taking the dot product with Eq. (7) and integrating over the length of the curved reference beam, we obtain the nonlinear functional $\mathrm{G}_{\mathrm{w}}(\hat{\varphi}, \boldsymbol{\Lambda}, h)$ corresponding to the virtual work principle, with the following form

$$
\begin{aligned}
\mathrm{G}_{\mathrm{w}}(\hat{\varphi}, \boldsymbol{\Lambda}, h)= & \int_{[0, L]}\left[\delta \hat{\varphi} \cdot\left(\hat{n},_{S}+\hat{n}_{\mathrm{p}}\right)\right. \\
& \left.+\delta \hat{\theta} \cdot\left(\hat{m},_{S}+\hat{\varphi},_{S} \times \hat{n}+\cdot \hat{m}_{\mathrm{p}}\right)\right] \mathrm{d} S .
\end{aligned}
$$

Integrating by parts for the $\hat{n},{ }_{S}$ and $\hat{m},{ }_{S}$ terms, one may obtains the spatial version of the weak form of the balance equations $[24,59]$ as

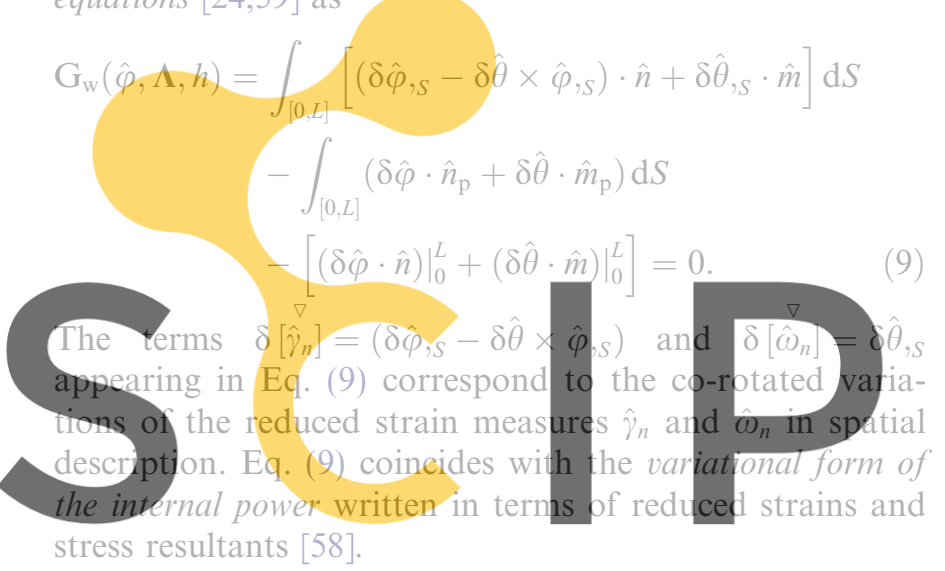

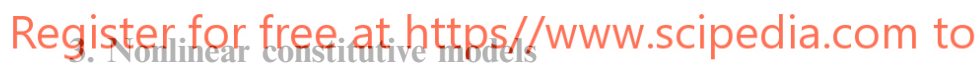

Frequently, the material properties have been assumed hyperelastic, isotropic and homogeneous [19,24,58,59] and, therefore, the reduced constitutive equations became very simple. Many times in engineering problems the interest is focused towards knowing the structural behavior beyond the linear elastic case and the modern design takes into account the capacity of the structure to resist loads with an adequate combination of strength, ductility and stiffness [18]. In this case, realistic studies involve constitutive nonlinearities as well as geometric effects. In this work, material points on the beam cross sections are considered as formed by a composite material corresponding to a homogeneous mixture of different simple components, each of them with its own constitutive law (see Fig. 3). The behavior of the composite is obtained by means of the mixing theory described in the following sections.

\subsection{Simple components}

Two kinds of nonlinear constitutive models for simple components are used in this work: the damage and plasticity models. They have been chosen due to the fact that combining different mechanical parameters and using the

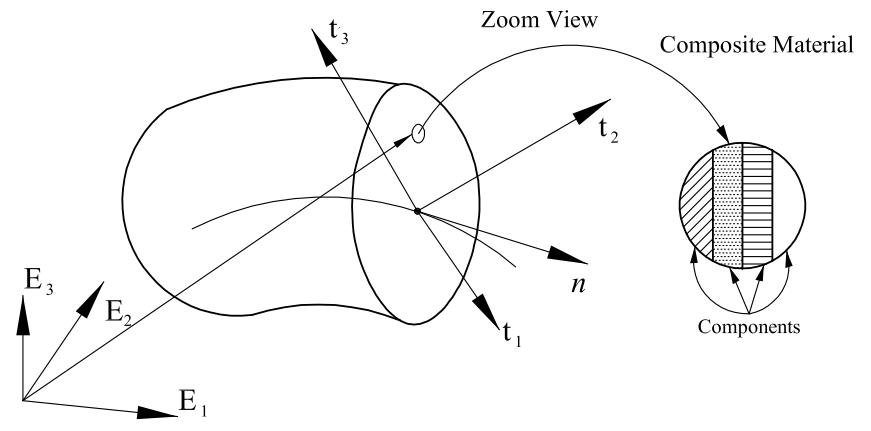

Fig. 3. Cross section showing the composite associated to a material point on the current configuration.

parallel version of the mixing rule for composites [46], a wide variety of mechanical behaviors can be reproduced e.g., concrete, fiber reinforced composites and masonry among others. The models presented for both, damage and plasticity, correspond to a particular cases of more general formulations, which can be consulted in $[6,21,42,44]$, but formulated in a way such that it is possible to include them in the geometrically exact formulation for beams previously described.

In this sense, considering the kinematics hypothesis of the beam model, we obtain that the components of any
spatial vector of tensor described the local frame $\left\{\hat{\mu}_{\}}\right\}$are
the same (in terms of their numerical values) as those of
their corresponding material forms described in the mate-
rial frame $\left\{\widehat{E}_{i}\right\}$ [58]. Therefore, the constitative models
are formulated in terms of the material form of the FPK stress and strain vectors, $\widehat{P}_{1}^{\mathrm{m}}$ and $\mathscr{E}_{n}$, respectively. The resulting components of the material form of the stres dowvalhapd thesversipn withqut the waternark as their spatial counterparts described in $\left\{\hat{t}_{i}\right\}$.

\subsubsection{Degrading materials: damage model}

The behavior of most of the degrading materials is presented attending to the fact that micro-fissuration in geomaterials occurs mainly due to the lack of cohesion between the particles, however a large amount of other processes are also involved as it can be consulted in [21]. Different micro-fissures connects each with others generating a distributed damage zone in the material. After a certain loading level, a fractured zone appears. The damage theory employed in this work is based on a special damage yielding function which differentiates the mechanical response for the tension or compression components of the stress vector. In this case, fissuration is interpreted as a local effect depending on the evolution of a set of parameters of the material and the corresponding evolution equations. The progress of the damage is based on the evolution of a scalar parameter which affects to all the components of the elastic constitutive tensor and in this sense, it constitutes an isotropic damage model [42].

From a purely mechanical point of view, a brief explanation about the numerical modeling of the damage can be provided by means of considering a representative 
volume of material and an arbitrary cut with normal $\hat{k}$, as it is shown in Fig. 4. In this Figure the undamaged area is $S_{k}$ and $\bar{S}_{k}$ is the effective area obtained subtracting the area of the defects from $S_{k}$. The damage variable associated to this surface is defined as $d=1-\left(\bar{S}_{k} / S_{k}\right)$, which measures the degradation level and is equal to zero before loading. As damage increases, the resisting area $\bar{S}_{k} \rightarrow 0$ and therefore, $d \rightarrow 1[6,21]$.

\section{Constitutive equation and mechanical dissipation}

In the case of thermally stable problems, this model has associated the following expression for the free energy density $\Psi$ in terms of the elastic free energy density $\Psi_{0}$ and the damage internal variable $d[39]$ :

$$
\Psi\left(\hat{\mathscr{E}}_{n}, d\right)=(1-d) \Psi_{0}=(1-d)\left(\frac{1}{2 \rho_{0}} \widehat{\mathscr{E}}_{n} \cdot\left(\mathscr{C}^{\mathrm{me}} \hat{\mathscr{E}}_{n}\right)\right),
$$

where $\widehat{\mathscr{E}}_{n}$ is the material form of the strain vector, $\rho_{0}$ is the mass density in the curyed reference configuration and $\mathscr{C}^{\text {me }}=\operatorname{Diag}\left[E_{0}, G_{0}, G_{0}\right]$ is the material form of the elastic constitutive tensor, with $E_{0}$ and $G_{0}$ the Young and shear undamaged elastic modulus.

In this case, considering that

\section{inequality for the mechanical diss}

form [34,39] can be written as
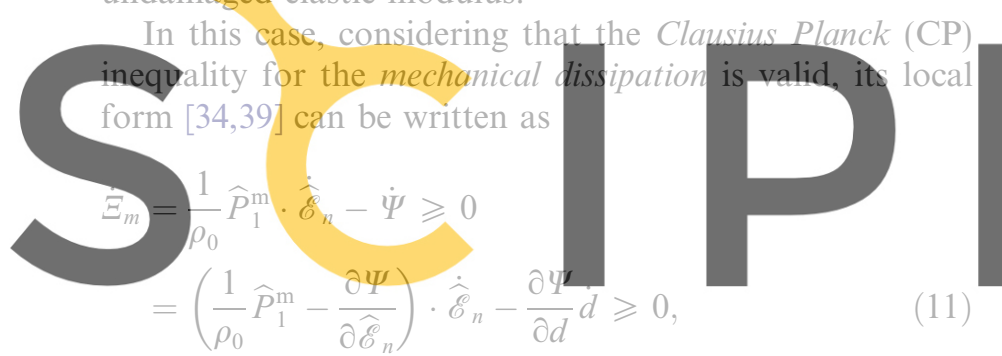

Register for free at https//www.scipedia.com to d where $\Xi_{m}$ is the dissipation rate.

For the unconditional fulfilment of the $\mathrm{CP}$ inequality and applying the Coleman's principle, we have that the arbitrary temporal variation of the free variable $\widehat{\mathscr{E}}_{n}$ must be null [34]. In this manner, the following constitutive relation for the material form of the FPK stress vector acting on each material point of the beam cross section is obtained:
$\widehat{P}_{1}^{\mathrm{m}}=(1-d) \mathscr{C}^{\mathrm{me}} \widehat{\mathscr{E}}_{n}=\mathscr{C}^{\mathrm{ms}} \widehat{\mathscr{E}}_{n}=(1-d) \widehat{P}_{01}^{\mathrm{m}}$,

where $\mathscr{C}^{\mathrm{ms}}=(1-d) \mathscr{C}^{\mathrm{me}}$ and $\widehat{P}_{01}^{\mathrm{m}}=\mathscr{C}^{\mathrm{me}} \widehat{\mathscr{E}}_{n}$ are the material form of the secant constitutive tensor and the elastic FPK stress vector, respectively. Inserting the result of Eq. (12) into (11) the following expression is obtained for the dissipation rate

$\dot{\Xi}_{m}=-\frac{\partial \Psi}{\partial d} \dot{d}=\Psi_{0} \dot{d} \geqslant 0$.

Eq. (12) shows that the FPK stress vector is obtained from its elastic (undamaged) counterpart by multiplying it by the degrading factor $(1-d)$. The internal state variable $d \in[0,1]$ measures the lack of secant stiffness of the material as it can be seen in Fig. 4. Moreover, Eq. (13) shows that the temporal evolution of the damage $\dot{d}$ is always positive due to the fact that $\Psi_{0} \geqslant 0$.

\section{Damage yield criterion}

By analogy with the developments presented in $[6,21,44]$, the damage yield criterion denoted by the scalar value $\mathscr{F}$ is defined as a function of the undamaged elastic free energy density and written in terms of the components of the material form of the undamaged principal stresses, $\widehat{P} \mathrm{~m}$, as

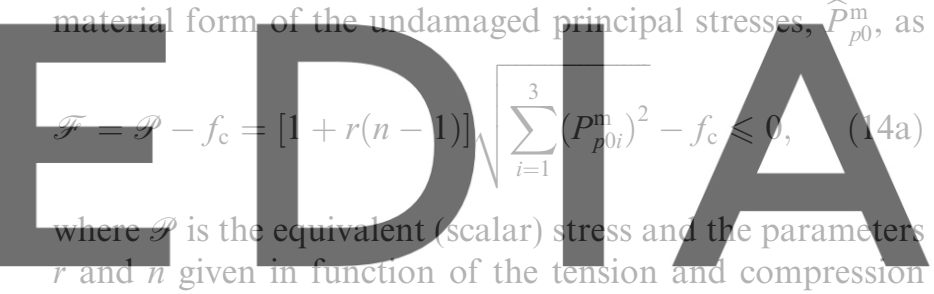

strengths $f_{\mathrm{c}}$ and $f_{\mathrm{t}}$ and the parts of the free energy density

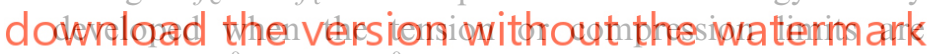
reached, $\left(\Psi_{\mathrm{t}}^{0}\right)_{L}$ and $\left(\Psi_{\mathrm{c}}^{0}\right)_{L}$, respectively, are defined as

$$
\begin{aligned}
& \left(\Psi_{t, c}^{0}\right)_{L}=\sum_{i=1}^{3} \frac{\left\langle \pm P_{p 0 i}^{\mathrm{m}}\right\rangle \mathscr{E}_{n i}}{2 \rho_{0}}, \quad \Psi_{L}^{0}=\left(\Psi_{\mathrm{t}}^{0}\right)_{L}+\left(\Psi_{\mathrm{c}}^{0}\right)_{L} \\
& f_{\mathrm{t}}=\left(2 \rho \Psi_{\mathrm{t}}^{0} E_{0}\right)_{L}^{\frac{1}{2}}, \quad f_{\mathrm{c}}=\left(2 \rho \Psi_{\mathrm{c}}^{0} E_{0}\right)_{L}^{\frac{1}{2}} \\
& n=\frac{f_{\mathrm{c}}}{f_{\mathrm{t}}}, \quad r=\frac{\sum_{i=1}^{3}\left\langle P_{p 0 i}^{\mathrm{m}}\right\rangle}{\sum_{i=1}^{3}\left|P_{p 0 i}^{\mathrm{m}}\right|}
\end{aligned}
$$
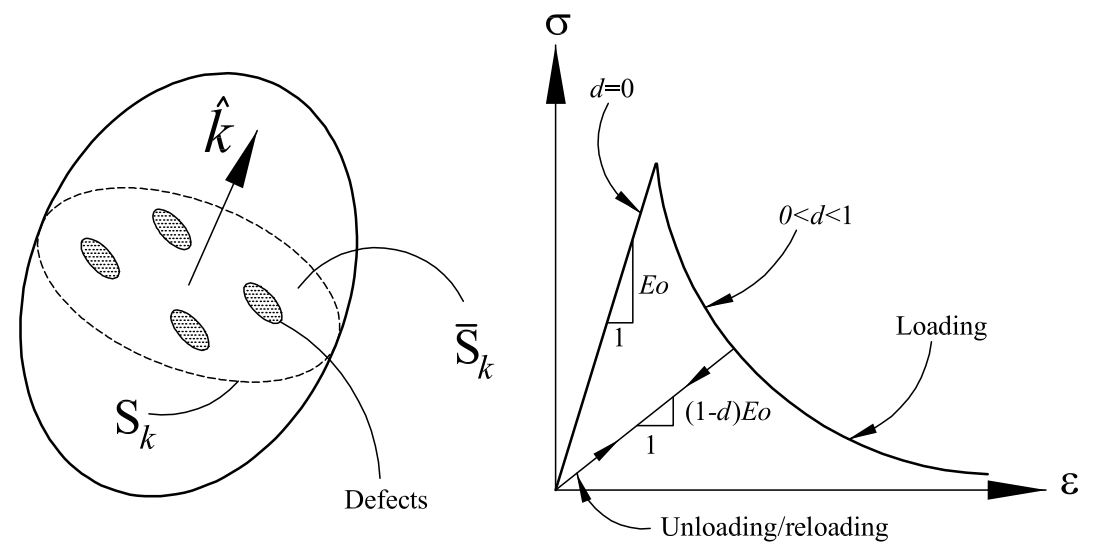

Fig. 4. Schematic representation of the damage model. 
where $|u|$ is the absolute value function and $\langle \pm u\rangle=$ $1 / 2(|u| \pm u)$ is the McAuley's function, $\forall u \in \mathbb{R}$. As shown in Ref. [21], other kind of damage yield criteria can be used in substitution of $\mathscr{P}$ e.g., Mohr-Coulomb, DruckerPrager, Von Mises etc., according to the mechanical behavior of the material.

A more general expression equivalent to that given in Eq. (14a) [6] is the following:

$\overline{\mathscr{F}}=\mathscr{G}(\mathscr{P})-\mathscr{G}\left(f_{\mathrm{c}}\right)$,

where $\mathscr{G}(\mathscr{P})$ is a scalar monotonic function to be defined in such way to ensure that the energy dissipated by the material on an specific integration point is limited to the specific energy fracture of the material [42].

Evolution of the damage variable

The evolution law for the internal damage variable $d$ is given by

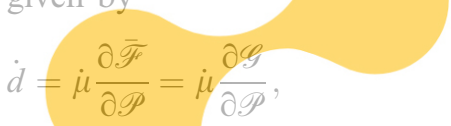

where $\dot{\mu} \geqslant 0$ is the damage consistency parameter. A damage yield condition $\overline{\mathscr{F}}=0$ and consistency condition $\mathscr{F}=0$ are defined analogously as in plasticity theory By one hand, the yield condition implies that $\mathscr{P} \rightarrow f_{\mathrm{c}} ; \quad \frac{\mathrm{d} \mathscr{G}(\mathscr{P})}{\mathrm{d} \mathscr{P}}=\frac{\mathrm{d} \mathscr{G}\left(f_{\mathrm{c}}\right)}{\mathrm{d} f_{\mathrm{c}}}$

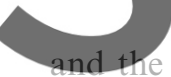
$\mathrm{d} f_{\mathrm{c}}$

definition of the damage variable (

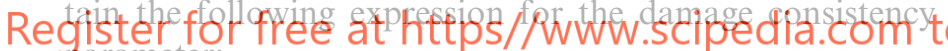
Saramele

$\mu=\dot{P}=\dot{f}_{\mathrm{c}}=\frac{\partial \mathscr{P}}{\partial \widehat{P}_{01}^{\mathrm{m}}} \cdot \dot{\widehat{P}}_{01}^{\mathrm{m}}=\frac{\partial \mathscr{P}}{\partial \widehat{P}_{01}^{\mathrm{m}}} \cdot \mathscr{C}^{\mathrm{me}} \dot{\widehat{\mathscr{O}}}_{n}$.

Details regarding the deduction of Eqs. (17) and (18) can be consulted in Refs. [6,21]. These results allow to rewrite Eqs. (13) and (16) as

$\dot{\Xi}_{m}=\Psi_{0}\left[\frac{\mathrm{d} \mathscr{G}}{\mathrm{d} \mathscr{P}} \frac{\partial \mathscr{P}}{\partial \widehat{P}_{01}^{\mathrm{m}}}\right] \cdot \mathscr{C}^{\mathrm{me}} \dot{\hat{\mathscr{E}}}_{n}$,

$\dot{d}=\frac{\mathrm{d} \mathscr{G}}{\mathrm{d} \mathscr{P}} \dot{P}$.

Finally, the Kuhn-Tucker relations: (a) $\dot{\mu} \geqslant 0$, (b) $\overline{\mathscr{F}} \leqslant 0$, (c) $\dot{\mu} \overline{\mathscr{F}}=0$, have to be employed to derive the unloadingreloading conditions i.e. if $\overline{\mathscr{F}}<0$ the condition (c) imposes $\dot{\mu}=0$, on the contrary, if $\dot{\mu}>0$ then $\mathscr{F}=0$.

\section{Definition of $\mathscr{G}$}

The following expression is employed for the function $\mathscr{G}$ of Eq. (15) [6,42]

$\mathscr{G}(\chi)=1-\frac{\overline{\mathscr{G}}(\chi)}{\chi}=1-\frac{\chi^{*}}{\chi} \mathrm{e}^{\kappa\left(1-\frac{\chi^{*}}{\chi}\right)}$, where the term $\overline{\mathscr{G}}(\chi)$ gives the initial yield stress for certain value of the scalar parameter $\chi=\chi^{*}$ and for $\chi \rightarrow \infty$ the final strength is zero. The parameter $\kappa$ of Eq. (20) is calibrated to obtain an amount of dissipated energy equal to the specific fracture energy of the material when all the deformation path is followed.

Integrating Eq. (11) for an uniaxial tension process with a monotonically increasing load, and considering that in this case the elastic free energy density can be written as $\Psi_{0}=\mathscr{P}^{2} /\left(2 n^{2} E_{0}\right)[6]$, it is possible to obtain that the total energy dissipated is [42]

$\Xi_{\mathrm{t}}^{\max }=\int_{\mathscr{P}^{*}}^{\infty} \underbrace{\frac{\mathscr{P}^{2}}{2 \rho_{0} n^{2} E^{0}}}_{\Psi_{0}} \mathrm{~d} \mathscr{G}(\mathscr{P})=\frac{\mathscr{P}^{* 2}}{2 \rho_{0} E^{0}}\left[\frac{1}{2}-\frac{1}{\kappa}\right]$.

Therefore, the following expression is obtained for $\kappa$

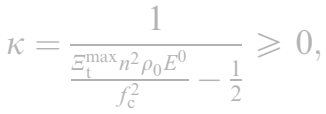

where it has been assumed that the equivalent stress tension $\mathscr{P}^{*}$ is equal to the initial damage stress $f_{\mathrm{c}}$.

The values of the maximum dissipation in tension $\Xi_{t}^{\max }$ is a material parameter equal to the dorresponding fracture energy density $g_{\mathrm{f}}^{d}=G_{\mathrm{f}}^{d} / l_{\mathrm{c}}$ $a^{d}$ $g_{\mathrm{f}}$, which is stic length of the fractured domain employed in the regu-

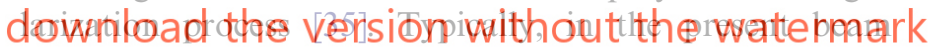
theory this length corresponds to the length of the fiber associated to an integration point on the beam cross section.

An identical procedure gives the fracture energy density $g_{\mathrm{c}}^{d}$ for a compression process yielding to the following expressions for $\kappa$

$\kappa=\frac{1}{\frac{\Xi_{\mathrm{c}}^{\max } \rho_{0} E^{0}}{f_{\mathrm{c}}^{2}}-\frac{1}{2}} \geqslant 0$.

Due to the fact that the value of $\kappa$ have to be the same for a compression or tension test, we have that $\Xi_{\mathrm{c}}^{\max }=\Xi_{\mathrm{t}}^{\max } n^{2}$.

\section{Tangent constitutive tensor}

Starting from Eq. (12) and after several algebraic manipulations which can be reviewed in $[6,21]$, we obtain that the material form of the tangent constitutive tensor $\mathscr{C}^{\mathrm{mt}}$ can be calculated as

$$
\begin{aligned}
\delta \widehat{P}_{1}^{\mathrm{m}} & =\mathscr{C}^{\mathrm{mt}} \delta \widehat{\mathscr{E}}_{n} \\
& =\left[(1-d) \mathbf{I}-\frac{\mathrm{d} \mathscr{G}}{\mathrm{d} \mathscr{P}} \widehat{P}_{01}^{\mathrm{m}} \otimes \frac{\partial \mathscr{P}}{\partial \widehat{P}_{01}^{\mathrm{m}}}\right] \mathscr{C}^{\mathrm{me}} \delta \widehat{\mathscr{E}}_{n},
\end{aligned}
$$


where $\mathbf{I}$ is the identity tensor. It is worth noting that $\mathscr{C}^{\mathrm{mt}}$ is non-symmetric and it depends on the elastic FPK stress vector.

A backward Euler scheme is used for the numerical integration of the constitutive damage model. The flow chart with the step-by-step algorithm used in numerical simulations is shown in Table 2.

\subsubsection{Plastic materials}

In case of materials which can undergo non-reversible deformations the plasticity model formulated in the material configuration is used for predicting their mechanical response. The model here presented is adequate to simulate the mechanical behavior of metallic and ceramic materials as well as geomaterials [45]. Assuming a thermally stable process and small elastic and finite plastic deformations, we have that the free energy density, $\Psi$, is given by the addition of the elastic and the plastic parts [35] as

$$
\Psi=\Psi^{\mathrm{e}}+\Psi^{\mathrm{P}}=\frac{1}{2 \rho_{0}}\left(\widehat{\mathscr{E}}_{n}^{\mathrm{e}} \cdot \mathscr{C}^{\mathrm{me}} \widehat{\mathscr{E}}_{n}^{\mathrm{e}}\right)+\Psi^{\mathrm{P}}\left(k_{\mathrm{p}}\right)
$$

where the $\widehat{\mathscr{E}}_{n}$ is the elastic strain calculated subtracting the plastic strain $\hat{\mathscr{E}}_{n}^{\mathrm{P}}$ from the total strain $\hat{\mathscr{E}}_{n}, \Psi^{\mathrm{e}}$ and $\Psi^{\mathrm{P}}$ are the

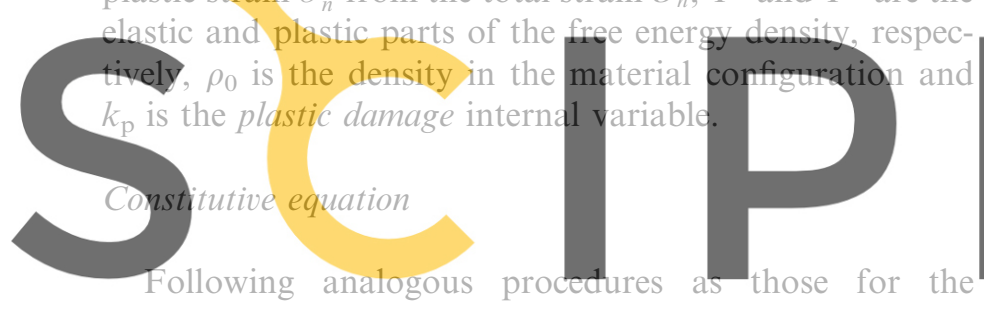

damage model i.e. employing the CP inequality and the

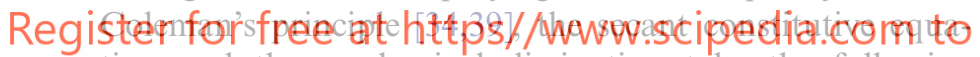

tion and the mechanical dissipation take the following forms:
$\widehat{P}_{1}^{\mathrm{m}}=\rho_{0} \frac{\partial \Psi\left(\widehat{\mathscr{E}}_{n}^{\mathrm{e}}, k_{\mathrm{p}}\right)}{\partial \widehat{\mathscr{E}}_{n}^{\mathrm{e}}}=\mathscr{C}^{\mathrm{ms}}\left(\widehat{\mathscr{E}}_{n}-\widehat{\mathscr{E}}_{n}^{\mathrm{P}}\right)=\mathscr{C}^{\mathrm{me}} \widehat{\mathscr{E}}_{n}^{\mathrm{e}}$

$\dot{\Xi}_{m}=\frac{\widehat{P}_{1}^{\mathrm{m}} \cdot \dot{\hat{\tilde{\boldsymbol{E}}}}_{n}^{\mathrm{P}}}{\rho_{0}}-\frac{\partial \Psi^{\mathrm{P}}}{\partial k_{\mathrm{p}}} \dot{k}_{\mathrm{p}} \geqslant 0$,

where the material description of the secant constitutive tensor $\mathscr{C}^{\mathrm{ms}}$ coincides with the elastic one $C^{\mathrm{me}}=$ Dia$\mathrm{g}\left[E_{0}, G_{0}, G_{0}\right]$. It is worth to note that Eqs. (27a) and (27b) constitute particular cases of a more general formulation of the so called coupled plastic damage models as it can be reviewed in [45].

\section{Yield and plastic potential functions}

Both, the yield function, $\mathscr{F}_{\text {p }}$ and plastic potential function, $\mathscr{G}_{\mathrm{p}}$, for the plasticity model, are formulated in terms of the material form of the FPK stress vector $\widehat{P}_{1}^{\mathrm{m}}$ and the plastic damage internal variable $k_{\mathrm{p}}$ as

$\mathscr{F}_{\mathrm{p}}\left(\widehat{P}_{1}^{\mathrm{m}}, k_{\mathrm{p}}\right)=\mathscr{P}_{\mathrm{p}}\left(\widehat{P}_{1}^{\mathrm{m}}\right)-f_{\mathrm{p}}\left(\widehat{P}_{1}^{\mathrm{m}}, k_{\mathrm{p}}\right)=0$,

(28a)

$\mathscr{G}_{\mathrm{p}}\left(\widehat{P}_{1}^{\mathrm{m}}, k_{\mathrm{p}}\right)=\mathscr{K}$

(28b)

Table 2

Flow chart for the damage model

(1) INPUT: material form of the strain vector $\widehat{\mathscr{E}}_{n}$ existing on a given integration point on the beam cross section

(2) Compute the material form of the elastic (undamaged) FPK stress vector, at the loading step $k$ and global iteration $j$ as

$$
\left(\widehat{P}_{01}^{\mathrm{m}}\right)_{j}^{(k)}=\mathscr{C}^{\mathrm{mt}}\left(\widehat{\mathscr{E}}_{n}\right)_{j}^{(k)}
$$

(3) Integration of the constitutive equation (Backward Euler scheme) Loop over the inner iterations: lth iteration

$$
\begin{aligned}
& \text { For } l=1 \quad \rightarrow \quad\left(\widehat{P}_{1}^{\mathrm{m}}\right)_{j}^{(k, 0)}=\left(\widehat{P}_{01}^{\mathrm{m}}\right)_{j}^{(k)} \\
& \text { (光) } \quad\left(\widehat{P}_{1}^{\mathrm{m}}\right)_{j}^{(k, l)}=\left(1-d_{j}^{(k, l)}\right)\left(\widehat{P}_{1}^{\mathrm{m}}\right)_{j}^{(k, 0)} \\
& \mathscr{P}_{j}^{(k, l)}=\mathscr{P}\left(\left(\widehat{P}_{1}^{\mathrm{m}}\right)_{j}^{(k, l)}\right) \quad \text { Eq. (14a) } \\
& \text { IF } \left.\overline{\mathscr{F}}_{(\mathscr{P}}^{(k, l)}, d_{j}^{(k, l)}\right) \leqslant 0 \rightarrow \text { no damage } \rightarrow \text { GOTO } 4 \\
& \text { ELSE } \rightarrow \text { Damage } \\
& (\Delta d)_{j}^{(k, l)}=G\left(\mathscr{P}_{j}^{(k, l)}\right)-d_{j}^{(k, l-1)} \text { Eq. (20) } \\
& d_{j}^{(k, l)}=(\Delta d)_{j}^{(k, l)}+d_{j}^{(k, l-1)} \\
& \left(\mathscr{C}^{\mathrm{mt} t}\right)_{j}^{(k, l)}=\mathscr{C}^{\mathrm{me}}\left[(1-d) \mathbf{I}-\frac{\mathrm{d} G}{\mathrm{~d} \mathscr{P}} \widehat{P}_{01}^{\mathrm{m}} \otimes \frac{\partial \mathscr{P}}{\partial \widehat{P}_{01}^{\mathrm{m}}}\right]_{j}^{(k, l)} \\
& l=l+1 \rightarrow \text { GO BACK TO }(\text { 梡 }
\end{aligned}
$$

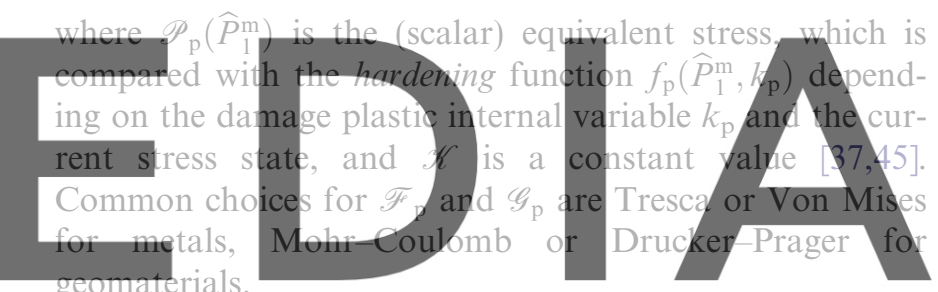

According to the evolution of the plastic damage vari-

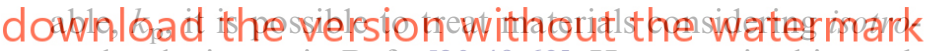
pic hardening as in Refs. [20,49,63]. However, in this work $k_{\mathrm{p}}$ constitutes a measure of the energy dissipated during the

(4) OUTPUT: Updated values of the FPK stress vector and tangent constitutive tensor i.e. $\left(\widehat{P}_{1}^{\mathrm{m}}\right)_{j}^{(k)}=\left(\widehat{P}_{1}^{\mathrm{m}}\right)_{j}^{(k, l)}$ and $\left(\mathscr{C}^{\mathrm{mt}}\right)_{j}^{(k)}=\left(\mathscr{C}^{\mathrm{mt}}\right)_{j}^{(k, l)}$ STOP 
plastic process and, therefore, it is well suited for materials with softening. In this case $k_{\mathrm{p}}$ is defined $[35,46]$ as

$g_{\mathrm{f}}^{\mathrm{P}}=\frac{G_{\mathrm{f}}^{\mathrm{P}}}{l_{\mathrm{c}}}=\int_{t=0}^{\infty} \widehat{P}_{1}^{\mathrm{m}} \cdot \dot{\mathscr{E}}_{n}^{\mathrm{P}} \mathrm{d} t$,

$0 \leqslant\left[k_{\mathrm{p}}=\frac{1}{g_{\mathrm{f}}^{\mathrm{P}}} \int_{t=0}^{t} \widehat{P}_{1}^{\mathrm{m}} \cdot \dot{\mathscr{E}}_{n}^{\mathrm{P}} \mathrm{d} t\right] \leqslant 1$,

where $G_{\mathrm{f}}^{\mathrm{P}}$ is the specific plastic fracture energy of the material in tension and $l_{\mathrm{c}}$ is the length of the fractured domain defined in analogous manner as for the damage model. The integral term in Eq. (29b) corresponds to the energy dissipated by means of plastic work and, therefore, $k_{\mathrm{p}}$ constitutes a measure of the part of the fracture energy that has been consumed during the deformation. Similarly, it is possible to define the normalized plastic damage variable for the case of a compressive test related with $g_{c}^{\mathrm{P}}$. If the plastic material is a part of a composite, the total fracture energy of the composite, which is a experimental quantity, is obtained as the sum of the fracture energy of the components, i.e., $G_{(f, e)}^{\mathrm{P}}=\sum_{i} G_{(f, e)}^{P(i)}$.

Evolution laws for the internal variables

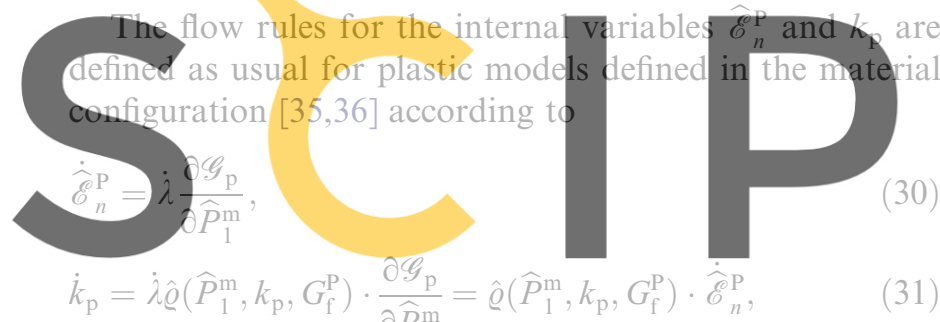

Register for free at https//www.scipedia.com to where $\lambda$ is the plastic consistency parameter and $\varrho$ is the following hardening vector $[35,45]$

$\dot{k}_{\mathrm{p}}=\left[\frac{r}{g_{\mathrm{f}}^{\mathrm{P}}}+\frac{1-r}{g_{\mathrm{c}}^{\mathrm{P}}}\right] \widehat{P}_{1}^{\mathrm{m}} \cdot \dot{\hat{\mathscr{E}}}_{n}^{\mathrm{P}}=\widehat{\varrho} \cdot \dot{\hat{\mathscr{E}}}_{n}^{\mathrm{P}}$.

The term $\widehat{P}_{1}^{\mathrm{m}} \cdot \dot{\hat{\mathscr{E}}}_{n}^{\mathrm{P}}$ is the plastic dissipation and $r$ is given in Eq. (14d). It is interesting to note that the proposed evolution rule allows to differentiate between tensile and compressive properties of the material, distributing the total plastic dissipation as weighted parts of the compressive and tensile fracture energy densities.

In what regards the hardening function of Eq. (28a), the following evolution equation has been proposed [37]:

$f_{\mathrm{p}}\left(\widehat{P}_{1}^{\mathrm{m}}, k_{\mathrm{p}}\right)=r \sigma_{\mathrm{t}}\left(k_{\mathrm{p}}\right)+(1-r) \sigma_{\mathrm{c}}\left(k_{\mathrm{p}}\right)$,

where $r$ has been defined in Eq. (14d) and the (scalar) functions $\sigma_{\mathrm{t}}\left(k_{\mathrm{p}}\right)$ and $\sigma_{\mathrm{c}}\left(k_{\mathrm{p}}\right)$ represent the evolution of the yielding threshold in uniaxial tension and compression tests, respectively. It is worth noting that in Eq. (33) a differentiated traction-compression behavior has been taken into account.

As it is a standard practice in plasticity, the loading/ unloading conditions are derived in the standard form from the Kuhn-Tucker relations formulated for problems with unilateral restrictions, i.e., (a) $\dot{\lambda} \geqslant 0$, (b) $\mathscr{F}_{\mathrm{p}} \leqslant 0$ and (c) $\dot{\lambda} \mathscr{F}_{\mathrm{p}}=0$.

By other hand, starting from the plastic consistency condition $\dot{\mathscr{F}}_{\mathrm{p}}=0$, and considering the flow rules of Eqs. (30) and (31), it is possible to deduce the explicit form of $\dot{\lambda}$ as $[45,46]$

$$
\dot{\lambda}=-\frac{\frac{\partial \mathscr{F}_{\mathrm{p}}}{\partial P_{1}^{\mathrm{m}}} \cdot\left(\mathscr{C}^{\mathrm{me}} \dot{\widehat{\mathscr{C}}}_{n}\right)}{\left\{\frac{\partial \mathscr{F}_{\mathrm{p}}}{\partial \widehat{P}_{1}^{\mathrm{m}}} \cdot\left(\mathscr{C}^{\mathrm{me}} \frac{\partial \mathscr{G}_{\mathrm{p}}}{\partial \widehat{P}_{1}^{\mathrm{m}}}\right)-\frac{\partial f_{\mathrm{p}}}{\partial k_{\mathrm{p}}} \hat{\varrho} \cdot \frac{\partial \mathscr{G}_{\mathrm{p}}}{\partial P_{1}^{\mathrm{m}}}\right\}} .
$$

\section{Tangent constitutive tensor}

The material form of the tangent constitutive tensor is calculated taking the time derivative of $\mathrm{Eq}$. (27a), considering the flow rule of Eq. (30) and replacing the plastic consistency parameter of Eq. (34), and after several algebraic manipulations, it is obtained as $[45,46]$

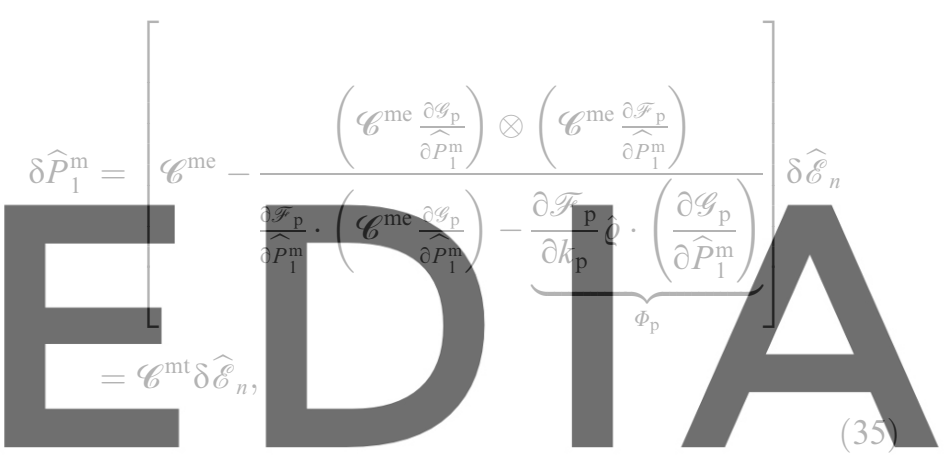

where $\Phi_{\mathrm{p}}$ is the so called hardening parameter.

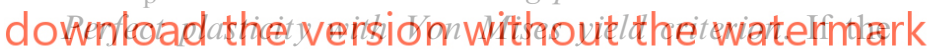
Von Mises criterion is chosen for the both the yielding and potential functions, equal tension/compression yielding thresholds are considered i.e., $n=1$ and $G_{\mathrm{f}}=G_{\mathrm{c}} \approx \infty$, one obtains that $k_{\mathrm{p}} \approx 0, \dot{k}_{\mathrm{p}} \approx 0$ and $f_{\mathrm{p}} \sim f_{\mathrm{c}}$ with $\sigma^{*}$ being the characteristic yielding threshold of the material, the following expressions are obtained

$$
\begin{aligned}
& \mathscr{F}_{\mathrm{p}}=\mathscr{P}_{\mathrm{p}}-f_{\mathrm{p}}=\sqrt{\widehat{P}_{1}^{\mathrm{m}} \cdot \mathscr{S} \widehat{P}_{1}^{\mathrm{m}}}-\sigma^{*} ; \quad \mathscr{S}=\operatorname{diag}[1,3,3], \\
& \frac{\partial \mathscr{F}_{\mathrm{p}}}{\partial \widehat{P}_{1}^{\mathrm{m}}}=\frac{\partial \mathscr{G}_{\mathrm{p}}}{\partial \widehat{P}_{1}^{\mathrm{m}}}=\frac{\mathscr{S} \widehat{P}_{1}^{\mathrm{m}}}{\mathscr{P}_{\mathrm{p}}}:=\hat{N}_{1}^{\mathrm{m}}, \\
& \dot{\lambda}=-\frac{\hat{N}_{1}^{\mathrm{m}} \cdot\left(\mathscr{C}^{\mathrm{me}} \dot{\hat{\mathscr{E}}}_{n}\right)}{\hat{N}_{1}^{\mathrm{m}} \cdot\left(\mathscr{C}^{\mathrm{me}} \hat{N}_{1}^{\mathrm{m}}\right)}, \quad \mathscr{C}^{\mathrm{mt}}=\mathscr{C}^{\mathrm{me}}-\frac{\left(\mathscr{C}^{\mathrm{me}} \hat{N}_{1}^{\mathrm{m}}\right) \otimes\left(\mathscr{C}^{\mathrm{me}} \hat{N}_{1}^{\mathrm{m}}\right)}{\hat{N}_{1}^{\mathrm{m}} \cdot\left(\mathscr{C}^{\mathrm{me}} \hat{N}_{1}^{\mathrm{m}}\right)} .
\end{aligned}
$$

In this particular case, more simple expressions are obtained as it can be seen in Eqs. (36a)-(36c) including a symmetric tangential tensor. Therefore, the perfect plasticity case can be considered as a limit case of the present formulation, for materials with infinite fracture energy.

The backward Euler scheme is used for the numerical integration of the constitutive plasticity model [45]. The flow chart with the step-by-step algorithm used in numerical simulations is shown in Table 3. 
Table 3

Flow chart for the plasticity model

(1) INPUT: material form of the strain vector $\widehat{\mathscr{E}}_{n}$ existing on a given integration point on the beam cross section

(2) Compute the material form of the predicted FPK stress vector, at the loading step $k$ and global iteration $j$ as

$$
\left(\widehat{P}_{1}^{\mathrm{m}}\right)_{j}^{(k)}=\mathscr{C}^{\mathrm{ms}}\left(\left(\widehat{\mathscr{E}}_{n}\right)_{j}^{(k)}-\left(\widehat{\mathscr{E}}_{n}^{\mathrm{P}}\right)_{(j-1)}^{(k)}\right)
$$

(3) Integration of the constitutive equation (Backward Euler scheme) Loop over the inner iterations: $l$ th iteration

$$
\begin{aligned}
& \text { For } l=1 \quad \rightarrow\left(\widehat{P}_{1}^{\mathrm{m}}\right)_{j}^{(k, 0)}=\left(\widehat{P}_{1}^{\mathrm{m}}\right)_{j}^{(k)}, \quad\left(\Delta \widehat{\mathscr{E}}_{n}^{\mathrm{P}}\right)_{j}^{(k, 0)}=0 \\
& \text { (灾) } \quad\left(\widehat{P}_{1}^{\mathrm{m}}\right)_{j}^{(k, l)}=\left(\widehat{P}_{1}^{\mathrm{m}}\right)_{j}^{(k, l-1)}-\mathscr{C}^{\mathrm{ms}}\left(\Delta \widehat{\mathscr{E}}_{n}^{\mathrm{P}}\right)_{j}^{(k, l-1)} \\
& \left(\mathscr{P}_{\mathrm{p}}\right)_{j}^{(k, l)}=\mathscr{P}_{\mathrm{p}}\left(\left(\widehat{P}_{1}^{\mathrm{m}}\right)_{j}^{(k, l)}\right) \\
& \text { IF } \mathscr{F}_{\mathrm{p}}\left(\mathscr{P}_{\mathrm{p}}, \widehat{E}_{n}^{\mathrm{P}}, k_{\mathrm{p}}\right)_{j}^{(k, l)} \leqslant 0 \rightarrow \text { elastic case } \rightarrow \text { GOTO } 4 \\
& \text { ELSE } \rightarrow \text { plastic case } \\
& \begin{array}{l}
\left(\Delta \widehat{\mathscr{E}}_{n}^{\mathrm{P}}\right)_{j}^{(k, l)}=(\Delta \lambda)_{j}^{(k, l)}\left(\frac{\partial \mathscr{G}_{\mathrm{p}}}{\partial \widehat{P}_{1}^{\mathrm{m}}}\right)_{j}^{(k, l)} \text { Eq. (34) } \\
\left(\widehat{\mathscr{E}}_{n}^{\mathrm{P}}\right)_{j}^{(k, l)}=\left(\widehat{\mathscr{E}}_{n}^{\mathrm{P}}\right)_{j}^{(k, l-1)}+\left(\Delta \widehat{\mathscr{E}}_{n}^{\mathrm{P}}\right)_{j}^{(k, l)} \\
\left(\Delta k_{\mathrm{p}}\right)_{j}^{(k, l)}=(\hat{\varrho})_{j}^{(k, l)} \cdot\left(\Delta \widehat{\mathscr{E}}_{n}^{\mathrm{P}}\right)_{j}^{(k, l)} \text { Eq. }(32) \\
\left(k_{\mathrm{p}}\right)_{j}^{(k, l)}=\left(k_{\mathrm{p}}\right)_{j}^{(k, l-1)}+\left(\Delta k_{\mathrm{p}}\right)_{j}^{(k, l)} \\
l=l+1 \rightarrow \text { GO BACK TO }(\preccurlyeq)
\end{array}
\end{aligned}
$$

(4) OUTPUT: Updated values of the FPK stress vector and tangent constitutive tensor i.e. $\left(\widehat{P}_{1}^{\mathrm{m}}\right)_{j}^{(k)}=\left(\widehat{P}_{1}^{\mathrm{m}}\right)_{j}^{(k, l)}$ and

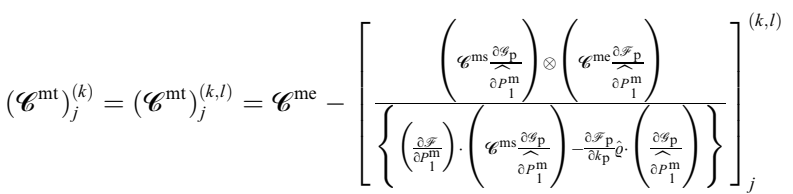

\subsection{Mixing theory for composites}

Each material point on the beam cross section is treated as a composite material according to the mixing theory $[11,45]$ considering the following assumptions: (i) Each composite has a finite number of components. ${ }^{3}$ (ii) Each component participates in the mechanical behavior according to its volumetric participation. (iii) All the components are subjected to the same strain field. Therefore, the interaction between all the components, defines the overall mechanical behavior of the composite at material point level.

The assumption (i) implies that the $N$ different components coexisting in a generic material point are subjected to the same material strain $\widehat{\mathscr{E}}_{n}$ and, therefore, we have the following closing equation:

$\widehat{\mathscr{E}}_{n} \equiv\left(\widehat{\mathscr{E}}_{n}\right)_{1}=\cdots=\left(\widehat{\mathscr{E}}_{n}\right)_{q}=\cdots=\left(\widehat{\mathscr{E}}_{n}\right)_{N}$

which imposes the strain compatibility between components. The free energy density of the composite, $\bar{\Psi}$, is written for the adiabatic case as

$\bar{\Psi}\left(\widehat{\mathscr{E}}_{n}, \alpha_{\mathrm{p}}\right) \equiv \sum_{q=1}^{N} k_{q} \Psi_{q}\left(\widehat{\mathscr{E}}_{n}, \alpha_{\mathrm{p}}\right)$

\footnotetext{
${ }^{3}$ Components are considered simple materials or compounding substances in the sense that only one constitutive law is used for describing their mechanical behavior.
}

where $\Psi_{q}\left(\widehat{\mathscr{E}}_{n}, \alpha_{\mathrm{p}}\right)(q=1 \ldots N)$ is the free energy density of the $q$ th compounding substance which depends on $p$ internal variables, $\left(\alpha_{\mathrm{p}}\right)_{q}$, and $k_{q}=V_{q} / V$, the quotient between the volume of the $q$ th component, $V_{q}$, and the total volume, $V$, is the volumetric fraction of the component and, therefore, $\sum_{q} k_{q}=1$ [11]. As it has been explained, in the present work only degrading and plastic materials are used as compounding substances, therefore, the values that the index $p$ can take, on the right side of Eq. (38), is limited to 1 for the degrading materials, (the damage variable $d_{q}$ ), and to 2 for the plastic ones (the plastic strain vector $(\widehat{\mathscr{E}} \mathrm{P}){ }_{q}$ and the plastic damage $\left.\left(k_{\mathrm{p}}\right)_{q}\right)$. In any case, a generic notation has been preferred by simplicity, even though it is necessary to have in mid that different substances have associated a different number of internal variables.

Starting from Eq. (38) and after applying the CP inequality and the Coleman's principle $[34,39]$, it is possible to obtain the material form of the FPK stress vector $\widehat{P}_{1}^{\mathrm{m}}$ and the mechanical dissipation $\dot{\bar{\Xi}}_{\mathrm{m}}$ for the composite in analogous way as for compounding subtances, i.e.

$$
\begin{gathered}
\widehat{P}_{1}^{\mathrm{m}} \equiv \bar{\rho}_{0} \frac{\partial \bar{\Psi}\left(\widehat{\mathscr{E}}_{n}, \alpha_{\mathrm{p}}\right)}{\partial \widehat{\mathscr{E}}_{n}}=\sum_{q=1}^{N} k_{q}\left(\rho_{0}\right)_{q} \frac{\partial \Psi_{q}\left(\widehat{\mathscr{E}}_{n}, \alpha_{\mathrm{p}}\right)}{\partial \widehat{\mathscr{E}}_{n}}=\sum_{q}^{N} k_{q}\left(\widehat{P}_{1}^{\mathrm{m}}\right)_{q}, \\
\dot{\bar{\Xi}}_{\mathrm{m}} \equiv-\sum_{q=1}^{N} k_{q}\left(\dot{\Xi}_{m}\right)_{q}=-\sum_{q=1}^{N} k_{q}\left[\sum_{m=1}^{p} \frac{\partial \Psi\left(\widehat{\mathscr{E}}_{n}, \alpha_{m}\right)}{\partial \alpha_{m}} \dot{\alpha}_{m}\right]_{q} \geqslant 0
\end{gathered}
$$


where $\widehat{P}_{1}^{\mathrm{m}}$ is obtained as a weighted sum, according to the volumetric fraction, of the material form of the stress vectors $\left(\widehat{P}_{1}^{\mathrm{m}}\right)_{q}$ corresponding to each one of the $N$ components. In the same manner is obtained the total mechanical dissipation, $\dot{\bar{\Xi}}_{m}$, i.e., considering the contribution of the $p$ internal variables of each one the compounding substances $\left(\dot{\Xi}_{m}\right)_{q}$.

The material form of the secant constitutive equation, the secant and tangent constitutive tensors, $\overline{\mathscr{C}}^{\mathrm{ms}}$ and $\overline{\mathscr{C}}^{\mathrm{mt}}$, for the composite are obtained as [45]

$\overline{\mathscr{C}}^{\mathrm{ms}} \equiv \sum_{q=1}^{N} k_{q}\left(\mathscr{C}^{\mathrm{ms}}\right)_{q} \rightarrow \widehat{P}_{1}^{\mathrm{m}}=\overline{\mathscr{C}}^{\mathrm{ms}}\left(\widehat{\mathscr{E}}_{n}-\widehat{\mathscr{E}}_{n}^{\mathrm{P}}\right)$,

$\widehat{\mathscr{E}}_{n}^{\mathrm{P}}=\sum_{q=1}^{N} k_{q}\left(\widehat{\mathscr{E}}_{n}^{\mathrm{P}}\right)_{q}$

$\delta \widehat{P}_{1}^{\mathrm{m}}=\overline{\mathscr{C}}^{\mathrm{mt}} \delta \widehat{\mathscr{E}}_{n}=\sum_{q=1}^{N} k_{q}\left(\mathscr{C}^{\mathrm{mt}}\right)_{q} \delta \widehat{\mathscr{E}}_{n}$,

where $\left(\mathscr{C}^{\mathrm{ms}}\right)_{q},\left(\mathscr{C}^{\mathrm{mt}}\right)_{q}$ and $\left(\widehat{\mathscr{E}}_{n} \mathrm{P}\right)_{q}$ are the material form of the secant and tangent constitutive tensors and the material form of the plastic strain vector of the $q$ th component, respectively.

It is worth to comment the meaning of $\bar{\rho}_{0}$ in Eq. (39a) and $\widehat{\mathscr{E}}_{n}^{\mathrm{P}}$ in Eq. (40b); they corresponds to the average value of the material forms of the density and the plastic strain vector of the composite and are obtained as a result of applying the mixing theory and, therefore, do not have a physical meaning. The secant constitutive tensors of the right side of Eq. (40a) correspond to the elastic one if a plastic material is used and to these given in Eq. (12) if a damage model is used for the $q$ th component.

Having calculated the material form of the FPK stress vector, the spatial form is obtained as $\widehat{P}_{1}=\boldsymbol{\Lambda} \widehat{P}_{1}^{\mathrm{m}}$. Stress resultant and couples are then calculated employing Eq. (6).

\section{Fiber reinforcements}

The mechanical behavior of some advanced composites are based on a main matrix component which is reinforced with oriented fibres, e.g., epoxy based materials with glass or carbon fibers or even reinforced concrete, where the steel reinforcing bars and stirrups can be seen as embedded reinforcing fibers. The mixing rule provides an appropriated framework to simulate these kind of composites [45], associating the one-dimensional version of the described constitutive laws to the reinforcements.

Due to the fact that the shape of the cross sections does not change during the motion, the incorporation of stirrups or other kind of transversal reinforcements is not allowed in the present formulation. However, it is possible to simulate the effect of this kind of reinforcement modifying the fracture energy and the limit stress of the matrix material. In the case of reinforced concrete, the shear resistance, ductility and strength increments due to confinement are simulated using this last simplified approach.

\section{Numerical implementation}

In order to obtain a Newton type numerical solution procedure, the linearized form of the weak form of Eq. (9) is required, which can be written as

$$
\mathscr{L}\left[\mathrm{G}_{\mathrm{w}}\left(\hat{\varphi}_{*}, \boldsymbol{\Lambda}_{*}, h\right)\right]=\mathrm{G}_{\mathrm{w}}\left(\hat{\varphi}_{*}, \boldsymbol{\Lambda}_{*}, h\right)+D \mathrm{G}_{\mathrm{w}}\left(\hat{\varphi}_{*}, \boldsymbol{\Lambda}_{*}, h\right) \cdot p,
$$

where $\mathscr{L}\left[\mathbf{G}_{\mathrm{w}}\left(\hat{\varphi}_{*}, \boldsymbol{\Lambda}_{*}, h\right)\right]$ is the linear part of the functional $\mathrm{G}_{\mathrm{w}}(\hat{\varphi}, \boldsymbol{\Lambda}, h)$ at the configuration defined by $(\hat{\varphi}, \boldsymbol{\Lambda})=$ $\left(\hat{\varphi}_{*}, \boldsymbol{\Lambda}_{*}\right)$ and $p \equiv(\Delta \hat{\varphi}, \Delta \hat{\theta}) \in \mathbb{R}^{3} \times T_{\Lambda}^{\mathrm{spa}}$ is an admissible variation. The term $\mathrm{G}_{\mathrm{w}}\left(\hat{\varphi}_{*}, \boldsymbol{\Lambda}_{*}, h\right)$ supplies the unbalanced force and the differential $D \mathrm{G}_{\mathrm{w}}\left(\hat{\varphi}_{*}, \boldsymbol{\Lambda}_{*}, h\right) \cdot p$, the tangential stiffness [59] which is calculated as

$$
\begin{aligned}
& D \mathrm{G}_{\mathrm{w}}\left(\hat{\varphi}_{*}, \boldsymbol{\Lambda}_{*}, h\right) \cdot p \\
& =\int_{[0, L]} \Delta\left(\left[\begin{array}{c}
\delta\left[\hat{\gamma}_{n *}\right]^{\nabla} \\
\delta\left[\hat{\omega}_{n *}\right]^{\nabla}
\end{array}\right]^{\mathrm{T}}\left[\begin{array}{c}
\hat{n}_{*} \\
\hat{m}_{*}
\end{array}\right]\right) \mathrm{d} S+\underbrace{\int_{[0, L]} \Delta\left(h^{\mathrm{T}}\left[\begin{array}{c}
\hat{n}_{p *} \\
\hat{m}_{p *}
\end{array}\right]\right) \mathrm{d} S}_{\mathrm{K}_{\mathbf{P} *}} \\
& =\int_{[0, L]}(\left[\begin{array}{c}
\Delta \delta\left[\hat{\gamma}_{n *}\right]^{\nabla} \\
\Delta \delta\left[\hat{\omega}_{n *}\right]^{\nabla}
\end{array}\right]^{\mathrm{T}}\left[\begin{array}{c}
\hat{n}_{*} \\
\hat{m}_{*}
\end{array}\right]+\underbrace{\left[\begin{array}{c}
\delta\left[\hat{\gamma}_{n *}\right]^{\nabla} \\
\delta\left[\hat{\omega}_{n *}\right]^{\nabla}
\end{array}\right]^{\mathrm{T}}}_{\left(\left[\mathbf{B}_{*}\right] h\right)^{\mathrm{T}}}\left[\begin{array}{c}
\Delta \hat{n}_{*} \\
\Delta \hat{m}_{*}
\end{array}\right]) \mathrm{d} S+\mathbf{K}_{\mathbf{P} *} \\
& =\int_{[0, L]}(\underbrace{\left[\begin{array}{cc}
0 & 0 \\
-\widetilde{\mathbf{n}}_{*}\left[\frac{\mathrm{d}}{\mathrm{d} S}\right] & 0
\end{array}\right]}_{\left[\mathbf{n}_{* *}\right]} p+h^{\left.\mathrm{T}\left[\mathbf{B}_{*}\right]^{\mathrm{T}}\left[\begin{array}{c}
\Delta \hat{n}_{*} \\
\Delta \hat{m}_{*}
\end{array}\right]\right) \mathrm{d} S+\mathbf{K}_{\mathbf{P} *},}
\end{aligned}
$$

where the subscript $*$ has been written to indicate that the involved quantities have to be evaluated at $(\hat{\varphi}, \boldsymbol{\Lambda})=$ $\left(\hat{\varphi}_{*}, \boldsymbol{\Lambda}_{*}\right)$, the skew-symmetric tensor $\widetilde{\mathbf{n}}_{*}$ is obtained from its axial vector $\hat{n}_{*}$, the operator $\left[\frac{\mathrm{d}}{\mathrm{d} S}\right]$ is defined as $\left[\frac{\mathrm{d}}{\mathrm{d} S}\right] \hat{v}=[\mathbf{I}] \hat{v}, \forall \hat{v} \in \mathbb{R}^{3}$, the operator $\left[\mathbf{n}_{S *}\right]$ contributes to the geometric part of the tangent stiffness, the term $\mathrm{K}_{\mathrm{P} *}$ corresponds to the part of the tangent stiffness which is dependent on the loading and the operator $\left[\mathbf{B}_{*}\right]$ relates the admissible variation $h$ and the co-rotated variation of the reduced strain vectors. Explicit expressions for $K_{P *}$ and $\left[\mathbf{B}_{*}\right]$ are omitted here and can be found in $[31,59]$.

The estimation of the linearized form of the sectional force and moment vectors appearing in Eq. (42) requires taking into account the linearized strain-stress relations existing between the material form of the FPK stress vector $\widehat{P}_{1}^{\mathrm{m}}$, obtained in each material point on the beam cross section according to Eq. (40c), and the material form of the strain vector $\widehat{\mathscr{E}}_{n}$ as follows

$$
\begin{aligned}
\Delta \widehat{P}_{1}^{\mathrm{m}} & =\overline{\mathscr{C}}^{\mathrm{mt}} \Delta \widehat{\mathscr{E}}_{n}, \\
\Delta\left[\widehat{P}_{1}\right] & =\boldsymbol{\Lambda} \Delta \widehat{P}_{1}^{\mathrm{m}}=\left(\boldsymbol{\Lambda} \overline{\mathscr{C}}^{\mathrm{mt}} \boldsymbol{\Lambda}^{\mathrm{T}}\right) \boldsymbol{\Lambda} \Delta \widehat{\mathscr{E}}_{n}=\overline{\mathscr{C}}^{\mathrm{st}} \Delta\left[\begin{array}{c}
\nabla \\
\hat{\varepsilon}_{n}
\end{array}\right] \\
& =\boldsymbol{\Lambda}\left(\Delta\left(\boldsymbol{\Lambda}^{\mathrm{T}} \widehat{P}_{1}\right)\right)=\Delta \widehat{P}_{1}-\Delta \widetilde{\theta} \widehat{P}_{1}, \\
\Delta \widehat{P}_{1} & =\Delta\left[\widehat{P}_{1}\right]-\widetilde{\mathbf{P}}_{1} \Delta \hat{\theta},
\end{aligned}
$$


where $\overline{\mathscr{C}}^{\mathrm{mt}}$ is the material forms of the tangential constitutive tensor for the composite which is obtained from Eq. (40c) and the corresponding spatial form is obtained as $\overline{\mathscr{C}}^{\text {st }}=\boldsymbol{\Lambda}^{\overline{\mathscr{C}}^{\mathrm{mt}}} \boldsymbol{\Lambda}^{\mathrm{T}}$. The skew-symmetric tensors $\boldsymbol{\Delta} \widetilde{\boldsymbol{\theta}}$ and $\widetilde{\mathbf{P}}_{1}$ are obtained from the linear increment in the rotation vector $\Delta \hat{\theta}$ and the FPK stress vector $\widehat{P}_{1}$, respectively.

Starting from the material form of Eq. (6), and employing Eq. (43a) it is possible to obtain linearized constitutive relation between the material form of stress resultant and stress couple vectors and the reduced strain vectors as

$$
\left\{\begin{array}{l}
\Delta \hat{n}^{\mathrm{m}} \\
\Delta \hat{m}^{\mathrm{m}}
\end{array}\right\}=\left[\begin{array}{ll}
\mathbf{C}_{11}^{\mathrm{mt}} & \boldsymbol{C}_{12}^{\mathrm{mt}} \\
\boldsymbol{C}_{21}^{\mathrm{mt}} & \mathbf{C}_{22}^{\mathrm{mt}}
\end{array}\right]\left\{\begin{array}{l}
\Delta \hat{\Gamma}_{n} \\
\Delta \hat{\Omega}_{n}
\end{array}\right\},
$$

where $\mathbf{C}_{p q}^{\mathrm{mt}},(p, q=1,2)$ are the material form of the reduced tangential constitutive tensors, which are calculated integrating over the beam cross section, as

$$
\begin{aligned}
& \mathbf{C}_{11}^{\mathrm{mt}}=\int_{\mathscr{A}} \overline{\mathscr{C}}^{\mathrm{mt}} \mathrm{d} \mathscr{A}, \quad \mathbf{C}_{12}^{\mathrm{mt}}=-\sum_{\beta=2}^{3} \int_{\mathscr{A}} \xi_{\beta} \overline{\mathscr{C}}^{\mathrm{mt}} \widetilde{\mathbf{E}}_{\beta} \mathrm{d} \mathscr{A}, \\
& \mathbf{C}_{21}^{\mathrm{mt}}=\int_{\mathscr{A}} \widetilde{\mathbf{Y}}^{\overline{\mathscr{C}}}{ }^{\mathrm{mt}} \mathrm{d} \mathscr{A}, \quad \mathbf{C}_{22}^{\mathrm{mt}}=-\sum_{\beta=2}^{3} \int_{\mathscr{A}} \xi_{\beta} \widetilde{\mathbf{T}}^{\overline{\mathscr{C}}}{ }^{\mathrm{mt}} \widetilde{\mathbf{E}}_{\beta} \mathrm{d} \mathscr{A},
\end{aligned}
$$

where $\widetilde{\mathbf{Y}}$ and $\widetilde{\mathbf{E}}_{\beta}$ are the skew-symmetric tensors obtained from the vectors $\hat{\Upsilon}=\Lambda^{\mathrm{T}}(\hat{x}-\hat{\varphi})$ and $\widehat{E}_{\beta}$, respectively. The corresponding spatial reduced tangential tensors are obtained as $\mathbf{C}_{p q}^{\mathrm{st}}=\boldsymbol{\Lambda} \mathbf{C}_{p q}^{\mathrm{mt}} \boldsymbol{\Lambda}^{\mathrm{T}}$, which are configuration dependent. The spatial form of the linearized relation of Eq. (44) can be obtained considering Eqs. (43b) and (43c) as

$$
\left\{\begin{array}{c}
\Delta \hat{n} \\
\Delta \hat{m}
\end{array}\right\}=\underbrace{\left[\begin{array}{ll}
\mathbf{C}_{11}^{\mathrm{st}} & \mathbf{C}_{12}^{\mathrm{st}} \\
\mathbf{C}_{21}^{\mathrm{st}} & \mathbf{C}_{22}^{\mathrm{st}}
\end{array}\right]}_{\left[\mathbf{C}^{\text {st }}\right]}\left\{\begin{array}{l}
\Delta\left[\hat{\gamma}_{n}\right]^{\nabla} \\
\Delta\left[\hat{\omega}_{n}\right]^{\nabla}
\end{array}\right\}-\underbrace{\left[\begin{array}{cc}
0 & \widetilde{\boldsymbol{n}} \\
0 & \widetilde{\boldsymbol{m}}
\end{array}\right]}_{[\widetilde{\mathbf{F}}]}\left\{\begin{array}{c}
\Delta \hat{\varphi} \\
\Delta \hat{\theta}
\end{array}\right\},
$$

where $\widetilde{\boldsymbol{m}}$ is the skew-symmetric tensor obtained from $\hat{m}$. Finally, Eq. (46) allows to rewrite Eq. (42) as

$$
\begin{aligned}
D \mathrm{G}_{\mathrm{W}}\left(\hat{\varphi}_{*}, \boldsymbol{\Lambda}_{*}, h\right) \cdot p= & \underbrace{\int_{[0, L]} h^{\mathrm{T}}\left[\mathbf{B}_{*}\right]^{\mathrm{T}}\left[\mathbf{C}_{*}^{\mathrm{st}}\right]\left[\mathbf{B}_{*}\right] p \mathrm{~d} S}_{\mathrm{K}_{\mathrm{M} *}} \\
& +\underbrace{\int_{[0, L]} h^{\mathrm{T}}\left(\left[\widetilde{\boldsymbol{n}}_{S *}\right]-\left[\mathbf{B}_{*}\right]^{\mathrm{T}}\left[\widetilde{\mathbf{F}}_{*}\right]\right) p \mathrm{~d} S}_{\mathrm{K}_{\mathrm{G} *}}+\mathrm{K}_{\mathrm{P} *}
\end{aligned}
$$

which allows to rewrite Eq. (41) as

$$
\mathscr{L}\left[\mathrm{G}_{\mathrm{w}}\left(\hat{\varphi}_{*}, \boldsymbol{\Lambda}_{*}, h\right)\right]=\mathrm{G}_{\mathrm{w}}\left(\hat{\varphi}_{*}, \boldsymbol{\Lambda}_{*}, h\right)+\mathrm{K}_{\mathrm{M} *}+\mathrm{K}_{\mathrm{G} *}+\mathrm{K}_{\mathrm{P} *},
$$

where $\mathrm{K}_{\mathrm{G} *}$ and $\mathrm{K}_{\mathrm{M} *}$, evaluated at the configuration $\left(\hat{\varphi}_{*}, \boldsymbol{\Lambda}_{*}\right)$, give the geometric and material parts of the tangent stiffness, which considers the inelastic behavior of the composite materials of the beam.

The solution of the discrete form of Eq. (48) by using the FE method follows identical procedures as those described in [59] for an iterative Newton-Raphson integration scheme and it will not be repeated here. The inclusion of material nonlinearity implies the addition of two additional integration loops, which are carried out at any material point on the cross section, but the rest of the integration procedure remains identical as for the case of elastic materials as it is explained in the next section.

\subsection{Cross sectional analysis}

The cross section analysis is carried out expanding each integration point on the beam axis in a set of integration points located on each fiber on cross section. In order to perform this operation, the beam cross section is meshed into a grid of quadrilaterals, each of them corresponding to a fiber oriented along the beam axis (see Fig. 5). The estimation of the average stress level existing on each quadrilateral is carried out by integrating the constitutive equations of the compounding materials of the composite associated to the corresponding quadrilateral and applying the mixing rule. The geometry of each quadrilateral is described by means of normalized bi-dimensional shapes functions and several integration points can be specified in order to estimate more precisely the value of a given function according to a selected integration rule. In the case of the average value of the material form of the FPK stress vector acting on a quadrilateral we have

$\widehat{P}_{1}^{\mathrm{m}}=\frac{1}{A_{\mathrm{c}}} \int \widehat{P}_{1}^{\mathrm{m}} \mathrm{d} A_{\mathrm{c}}=\frac{1}{A_{\mathrm{c}}} \sum_{p=1}^{N p} \sum_{q=1}^{N q} \widehat{P}_{1}^{\mathrm{m}}\left(y_{p}, z_{q}\right) J_{p q} W_{p q}$,

where $A_{\mathrm{c}}$ is the area of the quadrilateral, $N p$ and $N q$ are the number of integration points in the two directions of the normalized geometry of the quadrilateral, $\widehat{P}_{1}^{\mathrm{m}}\left(y_{p}, z_{q}\right)$ is the value of the FPK stress vector existing on a integration point with coordinates $\left(y_{p}, z_{q}\right)$ with respect to the reference beam axis, which is obtained from the corresponding material strain vector $\widehat{\mathscr{E}}_{n}$ using the constitutive laws and the mixing rule, $J_{p q}$ is the Jacobian of the transformation between normalized coordinates and cross sectional coordinates and $W_{p q}$ are the weighting factors. The coefficients of the tangent constitutive tensors can be estimated in an analogous manner as in Eq. (49) but replacing $\widehat{P}_{1}^{\mathrm{m}}\left(y_{p}, z_{q}\right)$ by $\overline{\mathscr{C}}^{\mathrm{mt}}\left(y_{p}, z_{q}\right)$. Finally, having obtained the stress level on each quadrilateral, the cross sectional forces and moments are obtained by means of the discrete form of Eq. (6) as

$\hat{n}^{\mathrm{m}}=\sum_{k=1}^{N \text { fiber }}\left(A_{\mathrm{c}}\right)_{k}\left(\widehat{P}_{1}^{\mathrm{m}}\right)_{k}, \quad \hat{m}^{\mathrm{m}}=\sum_{k=1}^{\text {Nfiber }}\left(A_{\mathrm{c}}\right)_{k} \hat{\ell}_{k} \times\left(\widehat{P}_{1}^{\mathrm{m}}\right)_{k}$,

were Nfiber is the number of quadrilaterals of the beam cross section, $\left(A_{\mathrm{c}}\right)_{k}$ is the area of the $k$ quadrilateral, $\left(\widehat{P}_{1}^{\mathrm{m}}\right)_{k}$ is the average value of the material form of the FPK stress vector and $\hat{\ell}_{k}=\left(0, y_{k}, z_{k}\right)$ are the coordinates of the gravity center of the $k$ th quadrilateral with respect to the local beam reference frame.

By applying the same procedure as in Eq. (50), we have that the material form of the reduced tangential tensors of Eqs. (45a) and (45b) are numerically estimated as 


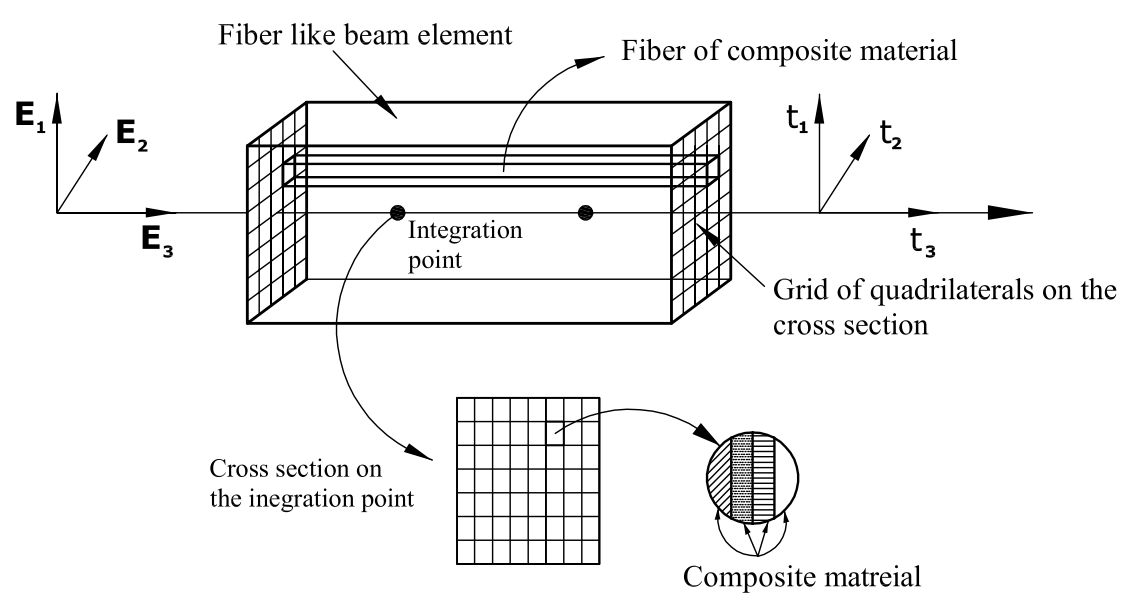

Fig. 5. Discrete fiber like model of the beam element.

$$
\begin{aligned}
& \mathbf{C}_{11}^{\mathrm{mt}}=\sum_{k=1}^{N \text { fiber }}\left(A_{\mathrm{c}}\right)_{k}\left(\overline{\mathscr{C}}^{\mathrm{mt}}\right)_{k}, \\
& \mathbf{C}_{12}^{\mathrm{mt}}=-\sum_{k=1}^{N \text { fiber }}\left(A_{\mathrm{c}}\right)_{k}\left(\overline{\mathscr{C}}^{\mathrm{mt}}\right)_{k}\left(y_{k} \widetilde{\boldsymbol{E}}_{2}+z_{k} \widetilde{\boldsymbol{E}}_{3}\right), \\
& \mathbf{C}_{21}^{\mathrm{mt}}=\sum_{k=1}^{N \text { fiber }}\left(A_{\mathrm{c}}\right)_{k} \widetilde{\boldsymbol{\ell}}_{k}\left(\overline{\mathscr{C}}^{\mathrm{mt}}\right)_{k}, \\
& \mathbf{C}_{22}^{\mathrm{mt}}=-\sum_{k=1}^{N \text { fiber }}\left(A_{\mathrm{c}}\right)_{k} \widetilde{\boldsymbol{\ell}}_{k}\left(\overline{\mathscr{C}}^{\mathrm{mt}}\right)_{k}\left(y_{k} \widetilde{\boldsymbol{E}}_{2}+z_{k} \widetilde{\boldsymbol{E}}_{3}\right),
\end{aligned}
$$

where $\widetilde{\ell}_{k}$ is the skew-symmetric tensor obtained from $\hat{\ell}_{k}$ and $\left(\overline{\mathscr{C}}^{\mathrm{mt}}\right)_{k}$ is the material form of the tangent constitutive tensor for the composite material of the $k$ th quadrilateral.
From the point of view of the numerical implementations in a given loading step and iteration, two additional integration loops are required. The first one is a loop over the quadrilaterals (or equivalently fibers). In this loop, having obtained the material form of the reduced strain measures $\hat{\Gamma}_{n}$ and $\hat{\Omega}_{n}$ (or equivalently $\widetilde{\mathbf{\Omega}}_{n}$ ), the strain measure $\widehat{\mathscr{E}}_{n}$ is calculated for each integration point on a quadrilateral. The second loop runs over each simple material associated to the composite of the quadrilateral. In this case, the FPK stress vector, $P_{1}^{\mathrm{m}}$, and the tangent constitutive relation, $\mathscr{C}^{\mathrm{mt}}$, are calculated for each component according to their specific constitutive equations; the behavior of the composite is recovered with the help of the mixing theory, summarized in Eqs. (39a) to (39b). Fig. 6 shows the flow

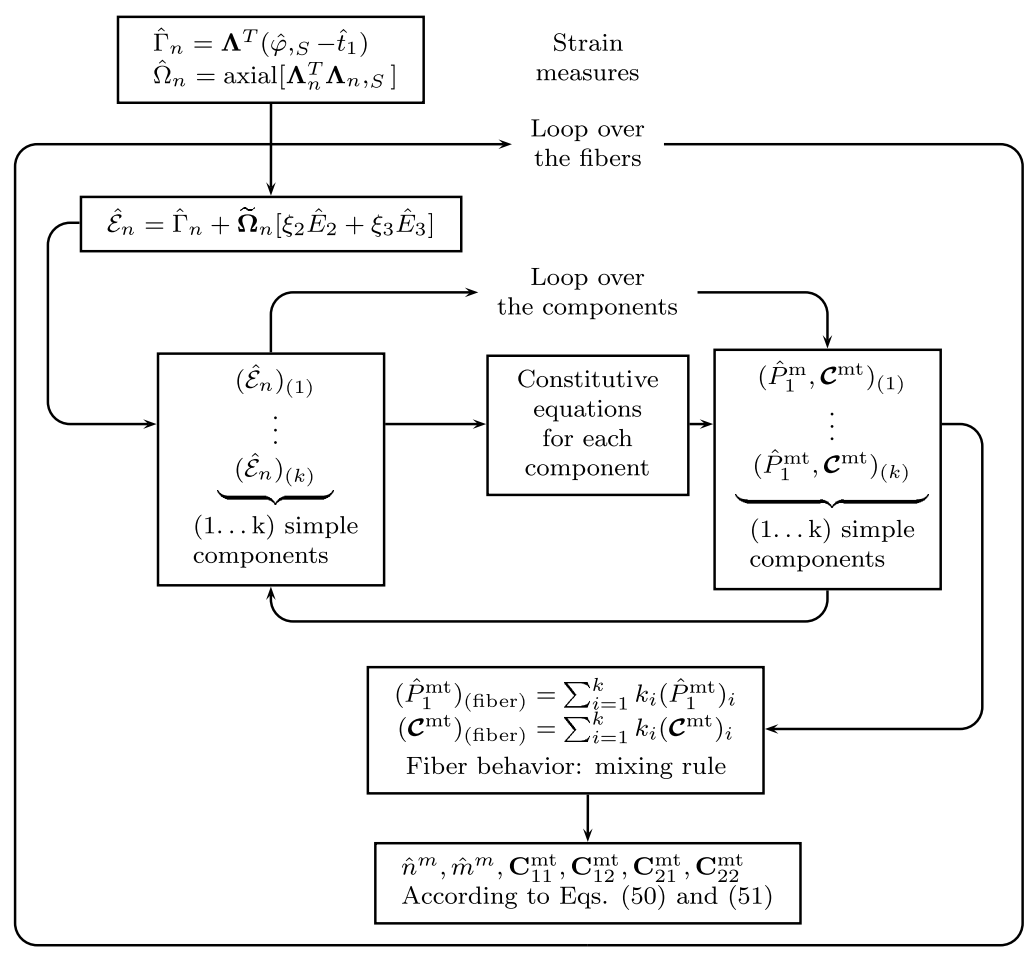

Fig. 6. Flow chart of the cross section integration procedure. 
chart of the cross sectional analysis providing the resultant cross sectional forces and moments. Finally, the discrete version of the spatial form of the reduced forces and moments, $\hat{n}=\boldsymbol{\Lambda} \hat{m}^{\mathrm{m}}$ and $\hat{m}=\boldsymbol{\Lambda} \hat{m}^{\mathrm{m}}$, and sectional tangent stiffness tensor $\mathbf{C}_{i j}^{\mathrm{st}}=\boldsymbol{\Lambda} \mathbf{C}_{i j}^{\mathrm{mt}} \boldsymbol{\Lambda}^{\mathrm{T}}(i, j=1,2)$ are calculated [31].

As it has been previously explained, the sectional behavior is obtained as the weighted sum of the contribution of the fibers, conversely to other works which develop global sectional integration methods [66,67]. Material nonlinearity, such as degradation or plasticity, is captured by means of the constitutive laws of the simple materials at each quadrilateral. The nonlinear relation between the reduced strain measures and cross sectional forces and moments are obtained from Eq. (50). Each section is associated to the volume of a part of the beam and, therefore, constitutive nonlinearity at beam element level is captured through the sectional analysis.

General shapes for cross sections can be analyzed by means of the proposed integration method. However, two limitations have to be considered: (i) Mechanical problems involving large deformations out of the cross sectional plane can not be reproduced due to the planarity of the cross sections considered in the kinematical assumptions. (ii) Mechanical equilibrium at element level does not implies mechanical equilibrium among fibers in the inelastic range due to the fact that the present beam model solves the constitutive equations for each fiber independently of the behavior of the contiguous ones.

\section{Damage index}

The estimation of damage indexes representative of the real remaining loading capacity of a structure has become a key issue in modern performance-based design approaches of civil engineering [32]. Several criteria have been defined for estimating the damage level of structures [21]; some of them are defined for the global behavior of the structure, others can be applied to individual members or subparts of the structure [12].

The damage index developed in this work is based on an analogy with the problem at micro-scale (constitutive) level. A measure of the damage level of a material point can be obtained as the ratio of the existing stress level, obtained applying the mixing rule, to its (undamaged) elastic counter part. Following this idea, it is possible to define the fictitious damage variable D as follows

$$
\begin{aligned}
& \sum_{i=1}^{3}\left|P_{1 i}^{\mathrm{m}}\right|=(1-\check{D}) \sum_{i=1}^{3}\left|P_{1 i 0}^{\mathrm{m}}\right|=(1-\check{D}) \sum_{i=1}^{3}\left|\left(\mathscr{C}^{\mathrm{m}} \widehat{\mathscr{E}}_{n}\right)_{i}\right|, \\
& \check{D}=1-\frac{\sum_{i=1}^{3}\left|P_{1 i}^{\mathrm{m}}\right|}{\sum_{i=1}^{3}\left|P_{1 i 0}^{\mathrm{m}}\right|}
\end{aligned}
$$

where $\left|P_{1 i}^{\mathrm{m}}\right|$ and $\left|P_{1 i 0}^{\mathrm{m}}\right|$ are the absolute values of the components of the existing and elastic stress vectors in material form, respectively. It is worth to note that $\check{D}$ considers any kind of stiffness degradation (damage, plasticity, etc.) at the material point level through the mixing rule and then it constitutes a measure of the remaining load carrying capacity. Initially, for low loading levels, the material remains elastic and $\check{D}=0$, but when the entire fracture energy of the material has been dissipated $\left|P_{1 i}^{\mathrm{m}}\right| \rightarrow 0$ and, therefore, $\check{D} \rightarrow 1$.

Eq. (52) can be extended to consider elements or even the whole structure by means of integrating the stresses over a finite volume of the structure. It allows defining the local and global damage indices as follows:

$\check{D}=1-\frac{\int_{V_{\mathrm{p}}}\left(\sum_{i}\left|P_{1 i}^{\mathrm{m}}\right|\right) \mathrm{d} V_{\mathrm{p}}}{\int_{V_{\mathrm{p}}}\left(\sum_{i}\left|P_{1 i 0}^{\mathrm{m}}\right|\right) \mathrm{d} V_{\mathrm{p}}}$,

where $V_{\mathrm{p}}$ is the volume of the part of the structure.

By one hand, the local/global damage index defined in Eq. (53) is a force-based criterium, which is able to discriminate the damage level assigned to a set of elements or to the whole structure, according to the manner in which they are loaded, in the same way as it has been explained in Ref. [21]. By the other hand, Eq. (53) is easily implemented in an standard FEM code without requiring extra memory storage or time consuming calculations.

\section{Numerical examples}

\subsection{Unrolling and rerolling of a circular beam}

This validation example considers the unrolling and rerolling of the elastic circular cantilever beam shown in Fig. 7. This example has been reported by Kapania and $\mathrm{Li}$ in Ref. [31] where four node initially curved FE elements are used. The case of an initially straight cantilever beam has been also reproduced in [24]. The initially circular beam has a radio $R=5 / \pi$, unitary square cross sectional area

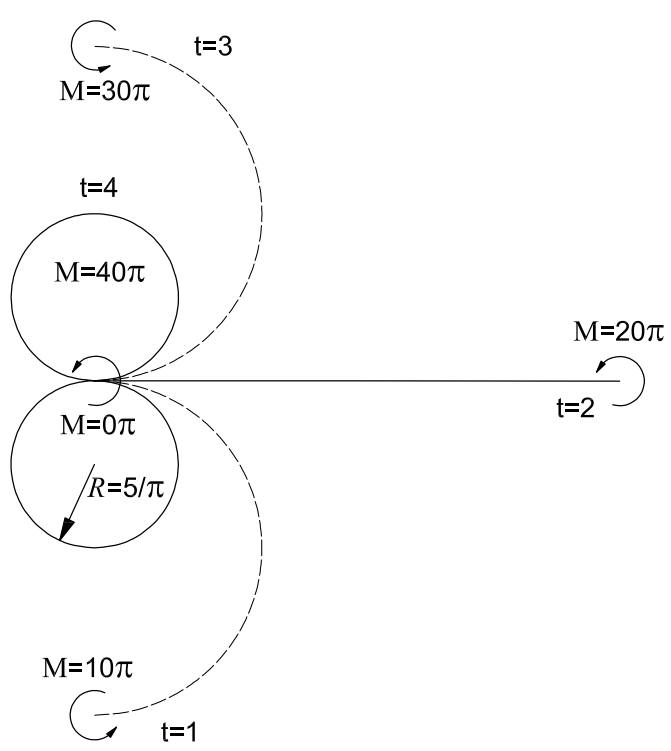

Fig. 7. Deformed configurations of the circular beam. 
and the following properties for the material: elastic modulus $E=1200$ and Poisson coefficient $v=0.0$. The FE mesh consist of ten equally spaced, quadratic, initially curved elements. An unitary bending moment $M$ is applied at the free end. Four loading steps are applied, each of them with a moment increment $\Delta M=10 \pi$. The convergence tolerance is $1 \times 10^{-7}$. The deformed configurations of the beam are shown in Fig. 7. The displacements of the free end for an applied moment of $10 \pi$ are 6.365 in the vertical direction and -0.001 in the horizontal direction. For an applied moment of $20 \pi$, the mentioned values are 0.003 and 9.998 , respectively, and are very close to those given in [31]. The number of iterations to reach the convergence in the first and second loading steps are 18 and 22 , respectively.

\subsection{Mesh independent response of a composite cantilever beam}

The RC cantilever beam shown in Fig. 8 is used to study if, regularizing the dissipated energy at constitutive level, it is possible to obtain a mesh independent response when including softening materials.

Forty increments of imposed displacements were applied in the $Y$ direction to obtain the capacity curve of the beam. Four meshes of 10, 20, 40 and 80 quadratic elements with the Gauss integration rule where considered in the simulations. The beam cross section was meshed into 20 equally spaced layers. The steel bars were included as a part of the composite material with a volumetric fraction corresponding to their contributing area to the total area of the layer where they are located. The mechanical properties of the concrete and steel are summarized in Table 4, where $E$ and $v$ are the elastic modulus and Poisson coefficient, respectively; $G_{\mathrm{f}}$ is the fracture energy, $f_{\mathrm{c}}$ is the ultimate compression limit and $n$ is the ratio of the compression to the tension yielding limits, according to Eq. (14c).

Fig. 9 shows the capacity curve relating the vertical reaction with the displacement of the free end. It is possible to see that the numerical responses converge to that corresponding to the model with the greater number of elements.

Further information can be obtained from the evolution of the local damage index at cross sectional level, which is shown in Fig. 10 for the four meshes and the loading steps 10, 25 and 40. In all the cases, strain localization occurs in the first element but, in the case of the mesh with 10 elements, localization occurs before than in the other cases
Table 4

Mechanical properties

\begin{tabular}{lclrrc}
\hline & $E(\mathrm{MPa})$ & $v(\mathrm{MPa})$ & \multicolumn{1}{c}{$f_{\mathrm{c}}$} & $n$ & $G_{\mathrm{f}}\left(\mathrm{N} \mathrm{mm}^{-2}\right)$ \\
\hline Concrete & 21000 & 0.20 & 25 & 8 & 1 \\
Steel & 200000 & 0.15 & 500 & 1 & 500 \\
\hline
\end{tabular}

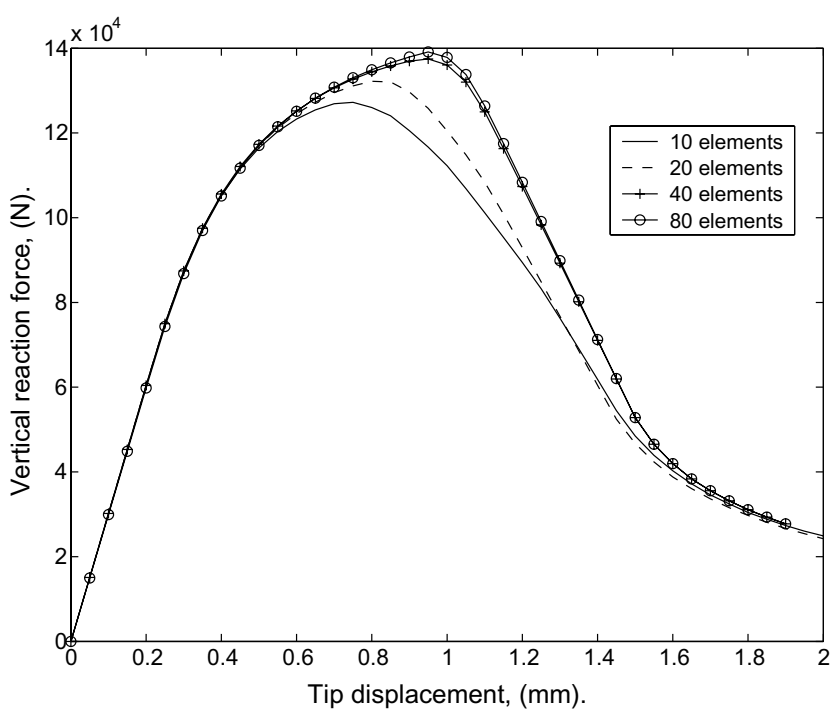

Fig. 9. Vertical reaction versus tip displacement.

and a worse redistribution of the damage is obtained, what can explain the differences observed in Fig. 9.

Finally, Fig. 11 shows the evolution of the global damage index which allows to appreciate the mesh independent response of the structure.

\subsection{Framed dome}

The elastic and plastic mechanical behavior of framed domes has been studied in several works. For example, in Ref. [17] domes formed by trusses are studied; in [49] initially straight beam elements are used to study the elastic plastic behavior of domes including isotropic and kinematic hardening; in [64] a co-rotational formulation for beam elements with lumped plasticity is presented; and in [65] a plastic hinge formulation assuming small strains and the Euler-Bernoulli hypothesis is employed for studying the nonlinear behavior of domes including the mechanical buckling and post critical loading paths.

In this example, the nonlinear mechanical behavior of the framed dome shown in Fig. 12 is studied with the objective of validating the proposed formulation in the inelastic

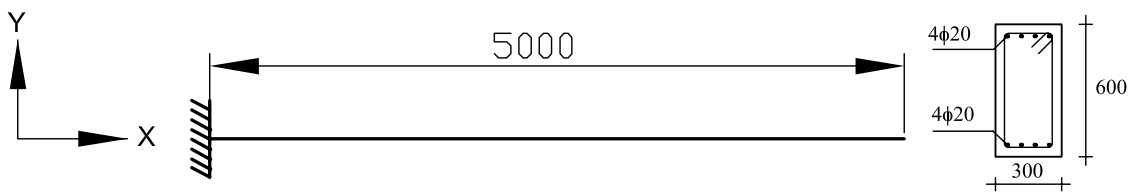

Fig. 8. RC cantilever beam. 


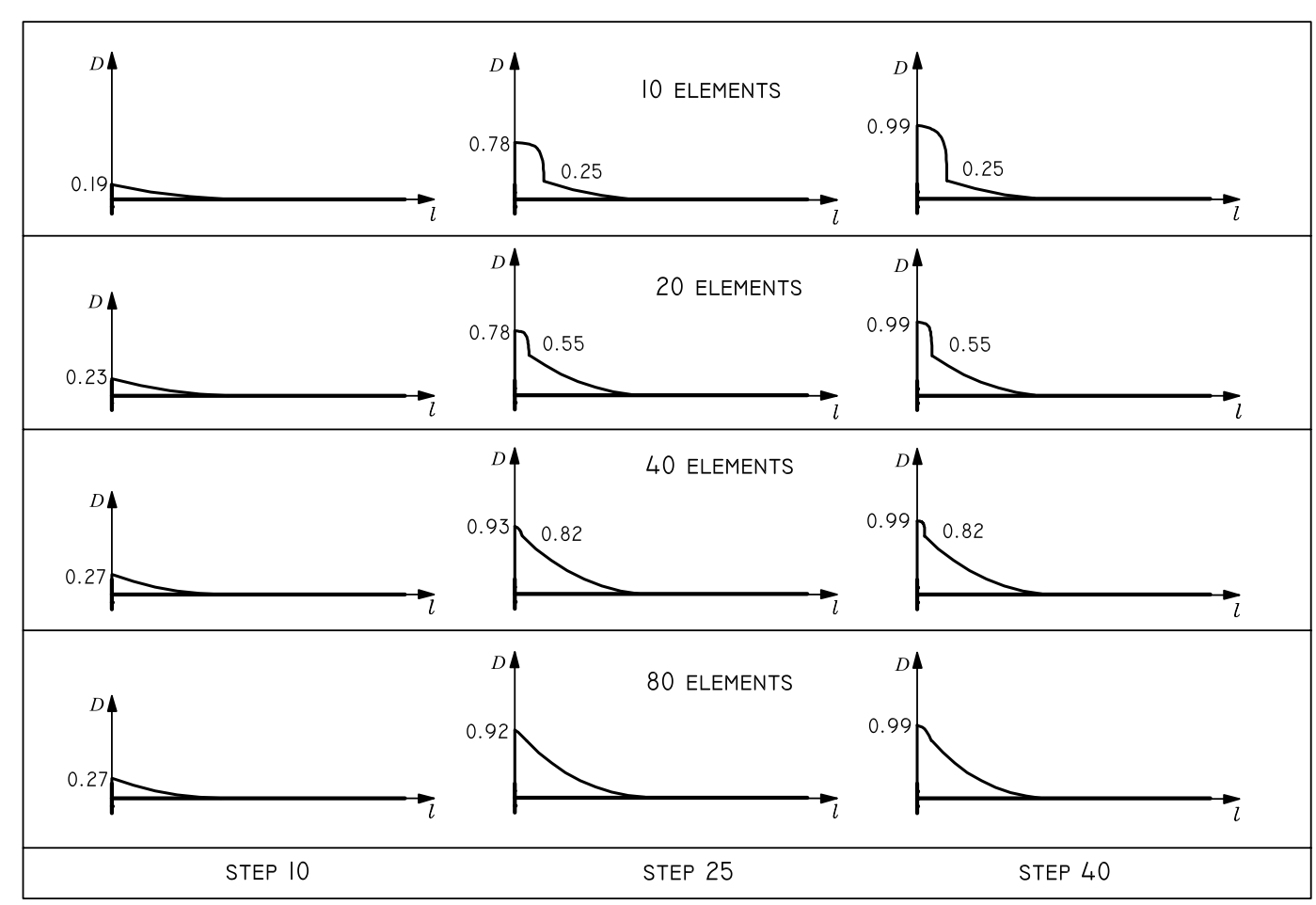

Fig. 10. Evolution of the local cross sectional damage index: Strain localization. The symbols $D$ and $l$ are the damage index and the length of the beam, respectively.

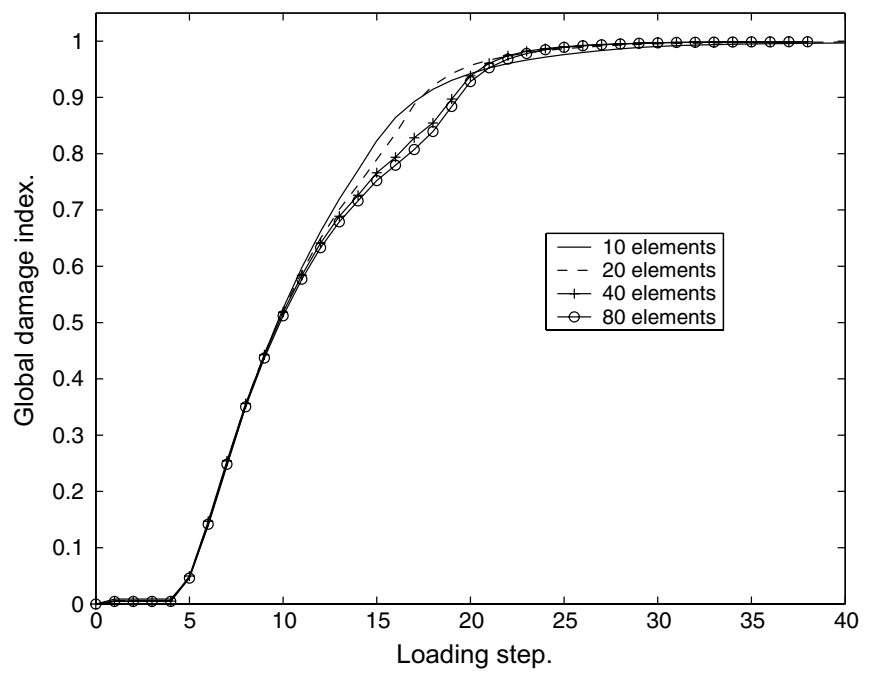

Fig. 11. Global damage index.

range. The linear elastic properties of the material are: elastic modulus $20700 \mathrm{MN} \mathrm{m}^{-2}$ and Poisson's coefficient 0.17. Three constitutive relations are employed: (1) Linear elastic; (2) Perfect plasticity $\left(G_{\mathrm{f}}=1 \times 10^{10} \mathrm{~N} \mathrm{~m}^{-2}\right)$ with associated Von Mises yield criterion and an elastic limit of $f_{\mathrm{c}}=80 \mathrm{~N} \mathrm{~m}^{-2}$; and (3) Damage model with equal tensile and compression limits, $n=1$, a fracture energy of $G_{f, c}=50 \mathrm{~N} \mathrm{~m}^{-2}$ and the same elastic limit as in case (2).

Three elements with two Gauss integration points are used for each structural member. A vertical point load of $P_{0}=123.8 \mathrm{~N}$ acting on the apex of the dome is applied and the displacement control technique is used in the simulations. Fig. 13 shows the deflection of the vertical apex in function of the loading factor $\lambda=P_{\mathrm{t}} / P_{0}\left(P_{\mathrm{t}}\right.$ is the current applied load) for the three constitutive relations. It is possible to see in Fig. 13 a good agreement with the results given by Park and Lee in Ref. [49] for the stable branch of the elastic loading factor-displacement responses. When comparing both results for the elastic plastic case, it is possible to see a good agreement for the elastic limit of the structure; however, when deformation grows, the differences can reach $30 \%$ for the predicted value of the load carrying capacity of the dome. Moreover, the curve corresponding to the damage model has been added to Fig. 13. In both cases, when inelastic constitutive relations are employed, the curve of the global structural response shows a snap-through which couples constitutive and geometric effects.

\subsection{Nonlinear response of a $45^{\circ}$ cantilever bend}

This example performs the coupled geometrically and constitutive nonlinear analysis of a cantilever bend placed in the horizontal $X-Y$ plane, with a vertical load $F$ applied at the free end, as shown in Fig. 14. The radius of the bend has $100 \mathrm{~mm}$ with unitary cross section. The linear elastic case of this example involves large 3D rotations and an initially curved geometry; therefore, it has been considered in several works as a good validation test $[24,31,59]$. The mechanical properties for the elastic case are an elastic modulus of $1 \times 10^{7} \mathrm{~N} \mathrm{~mm}^{-2}$ and a shear modulus of 

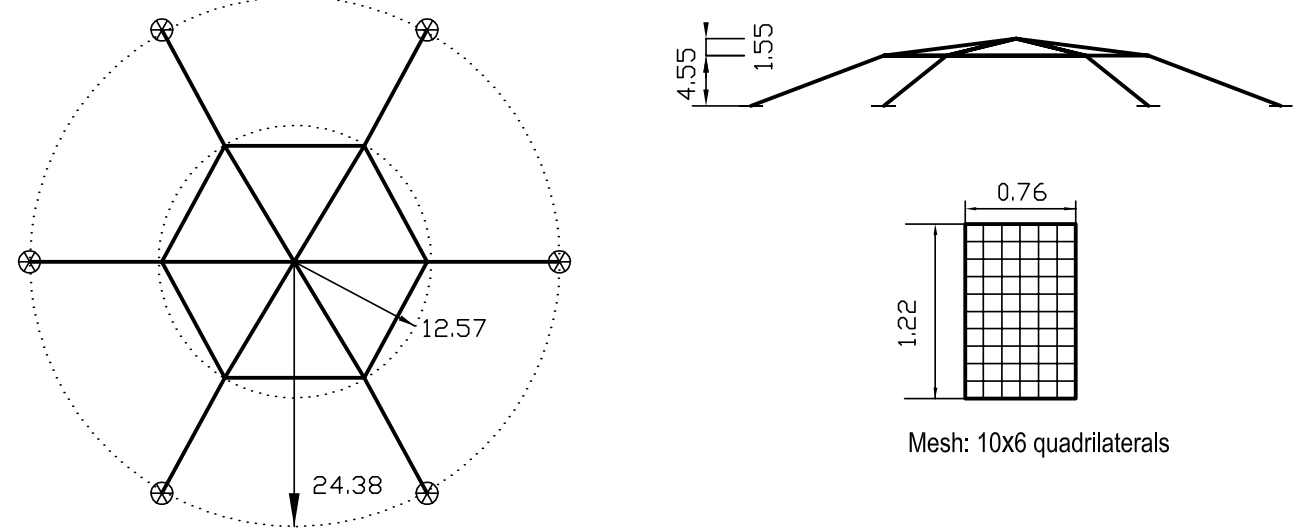

Mesh: $10 \times 6$ quadrilaterals

Fig. 12. Framed dome and detail of the cross sectional mesh.

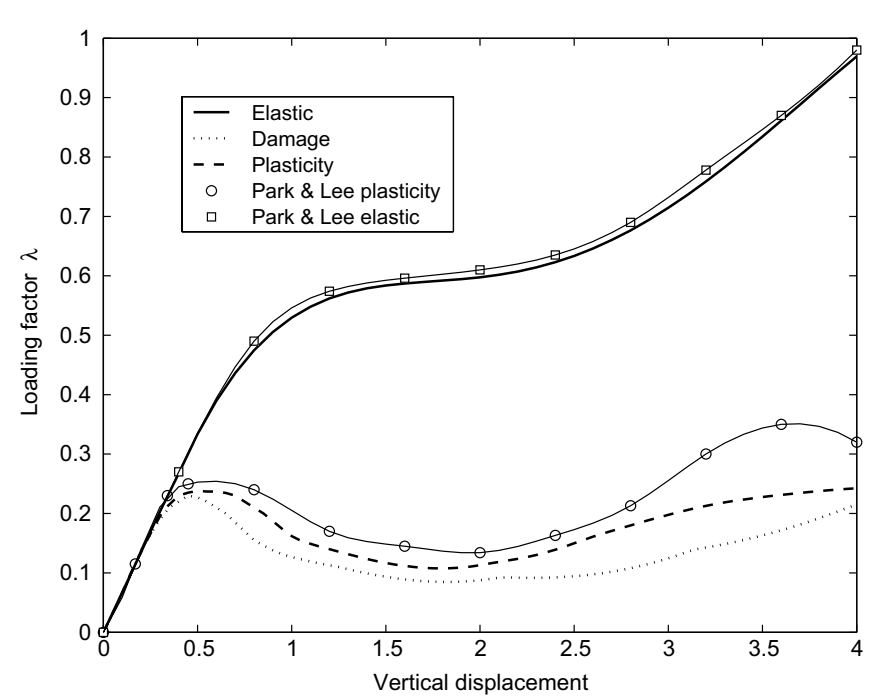

Fig. 13. Loading factor-displacement curve of the vertical apex of the dome.

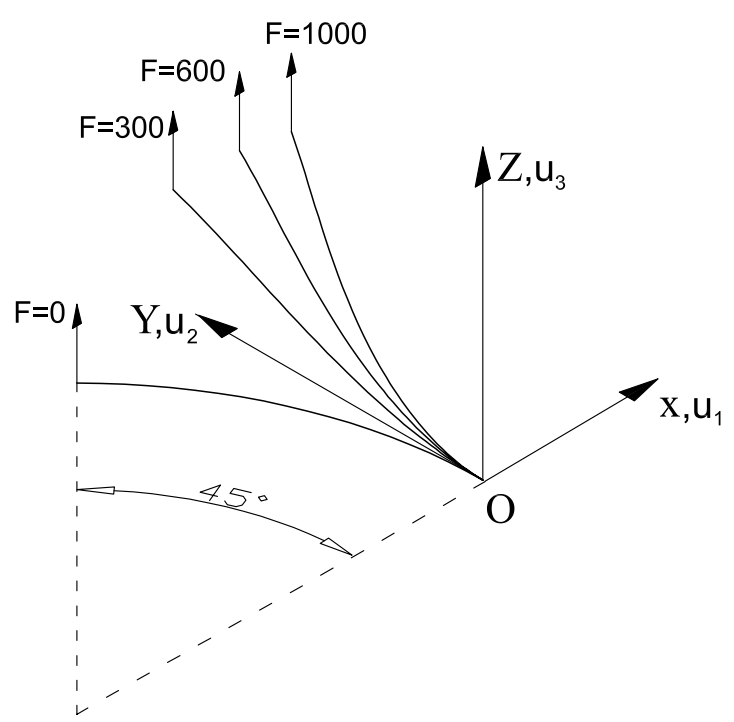

Fig. 14. Initial geometry and some examples of the deformed configurations for the linear elastic case of the $45^{\circ}$ cantilever bend.
$5 \times 10^{6} \mathrm{~N} \mathrm{~mm}^{-2}$. Four quadratic initially curved elements are used in the FE discretization with two Gauss integration points per element. Solutions are obtained by using thirty equal load increments of $100 \mathrm{~N}$. The history of the tip displacements is shown in Fig. 15. The tip displacements for an applied load of $600 \mathrm{~N}$ are: $U_{1}=13.56 \mathrm{~mm}$. $U_{2}=-23.81 \mathrm{~mm}$ and $U_{3}=53.51 \mathrm{~mm}$ (see Fig. 14) which are values close to those obtained by other authors [31].

The coupled geometric and constitutive nonlinear response of the structure was obtained for three materials: (i) Elastic-plastic (Von Mises yield criterion, associated flow rule, a fracture energy of $G_{\mathrm{f}}=1 \times 10^{10} \mathrm{~N} \mathrm{~mm}^{-2}$, and a tension to compression ratio $n=1$ ). (ii) Degrading material $\left(n=1, G_{\mathrm{f}}=5 \times 10^{4} \mathrm{~N} \mathrm{~mm}^{-2}\right)$ and (iii) a composite formed by equal parts of the materials (i) and (ii). In all the cases, the elastic limit is taken $f_{\mathrm{c}}=7 \times 10^{4} \mathrm{~N} \mathrm{~mm}^{-2}$.

The beam cross section was meshed into a grid of $10 \times 10$ quadrilaterals with one integration point per fiber. A set of 35 imposed displacements of $2 \mathrm{~mm}$ was applied. The convergence tolerance was taken equal to $10^{-4}$ for residual forces and displacements. Fig. 16 shows the results obtained from the numerical simulations for tip displacements superposed to the elastic response. It is possible to observe in this figure that: (1) The elastic plastic case converges to a fixed value of $274 \mathrm{~N}$ for the vertical reaction after the redistribution of the damage has occurred along the beam length, which can be considered the final stage in the formation of a plastic hinge in the structure. (2) In

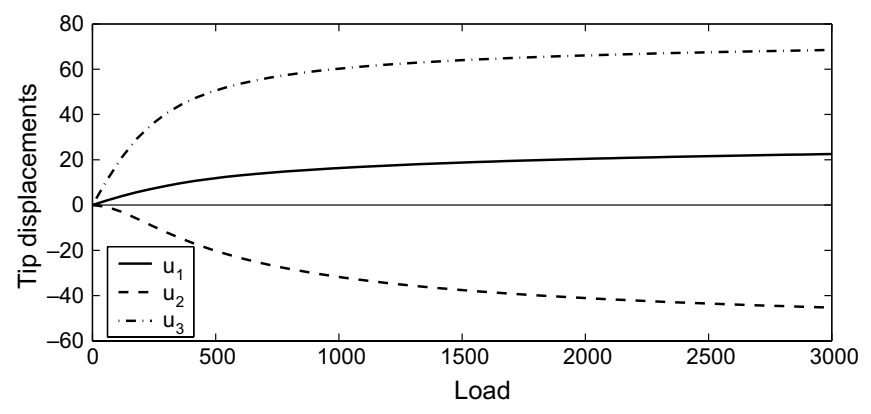

Fig. 15. Different components of the tip displacement. 


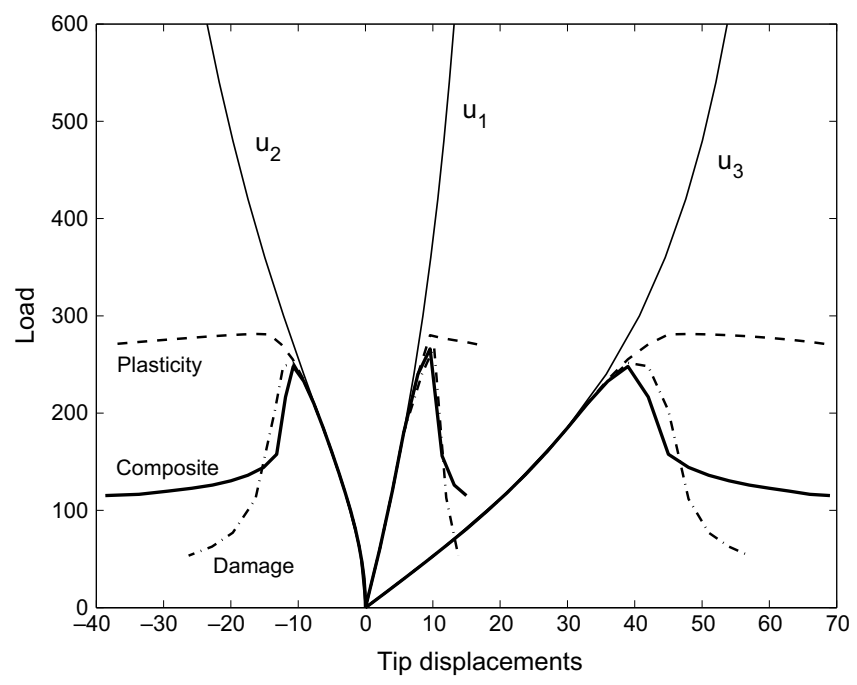

Fig. 16. Different components of the tip displacement.

the case of the degrading material, the analyze were stopped in the loading step 29 due to lack of convergency with an evident loss in the load carrying capacity. (3) The response of the composite materials show two phases: the first one corresponds to the degradation of the damaging phase; during the second one the vertical reaction is stabilized in a value equal to $112 \mathrm{~N}$, which is due to the mechanical response of the plastic phase.

In all the cases, a great amount of iterations were required to obtain convergence $(>50$ in the softening phase). However, in the case of the material (ii) the analyze were finalized after 300 iterations due to the fact that the development of axial forces in the deformed configuration literally cuts the beam for vertical displacements beyond $57 \mathrm{~mm}$.

\subsection{Nonlinear analysis of a right angle frame}

The right angle frame of Fig. 17 is subjected to a concentrated out of plane load $P=0.3 \mathrm{~N}$ acting on the middle of the span of one of the two members of length $L=100 \mathrm{~mm}$. Forty equally sized loading steps have been used. Each member is modeled using four quadratic elements with

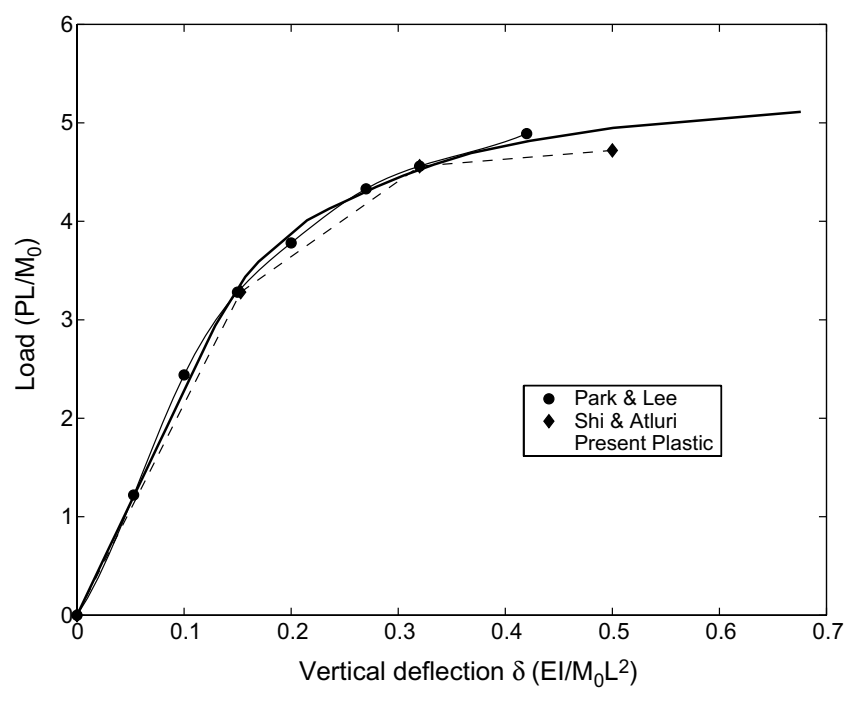

Fig. 18. Load-deflection curve of the right bent.

two Gauss integration points. The square beam cross sections with a side length $a=3 \mathrm{~mm}$ are meshed into a grid of $4 \times 4$ quadrilaterals with four integration points per fiber. The convergence tolerance is taken as $1 \times 10^{-4}$.

The constitutive and geometric nonlinear response of the frame is computed for four different materials with the same elastic modulus $E=720 \mathrm{~N} \mathrm{~mm}^{-2}$, Poisson coefficient $v=0.3$ and yielding threshold $f_{\mathrm{c}}=1 \mathrm{~N} \mathrm{~mm}^{-2}$. The other characteristics of the materials are: (i) associated Von Mises plasticity with a compression to tension ratio $n=1$ and fracture energy $G_{\mathrm{f}}=1 \times 10^{5} \mathrm{~N} \mathrm{~mm}^{-2}$; (ii) a damage model with $n=2, G_{\mathrm{f}}=0.1 \mathrm{~N} \mathrm{~mm}^{-2}$; (iii) a composite with a $20 \%$ of (i) and $80 \%$ of (iii). (iv) a composite with a $10 \%$ of (i) a $80 \%$ of (ii) and a $10 \%$ of a material having only linear elastic properties.

Fig. 18 shows a comparison between the results obtained for the applied force versus the vertical deflection of the point $A$ (see Fig. 17) for the material (i) and the results given in Refs. [49,57]. The results shown in Fig. 18 are normalized considering that $I$ is the inertia of the square cross section and $M_{0}=a^{3} f_{\mathrm{c}} / 6$. A good agreement with the results obtained in the mentioned references is obtained.

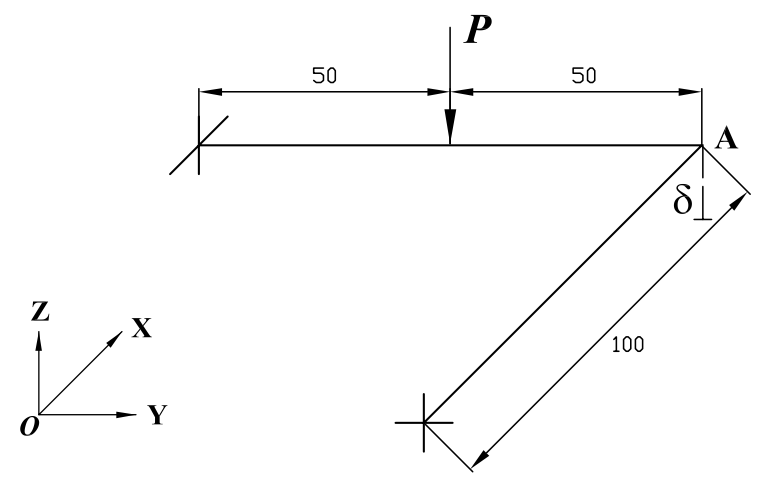

Fig. 17. Right bent.

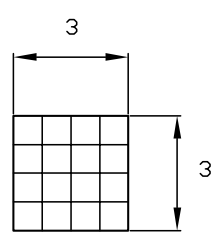

Cross section mesh 

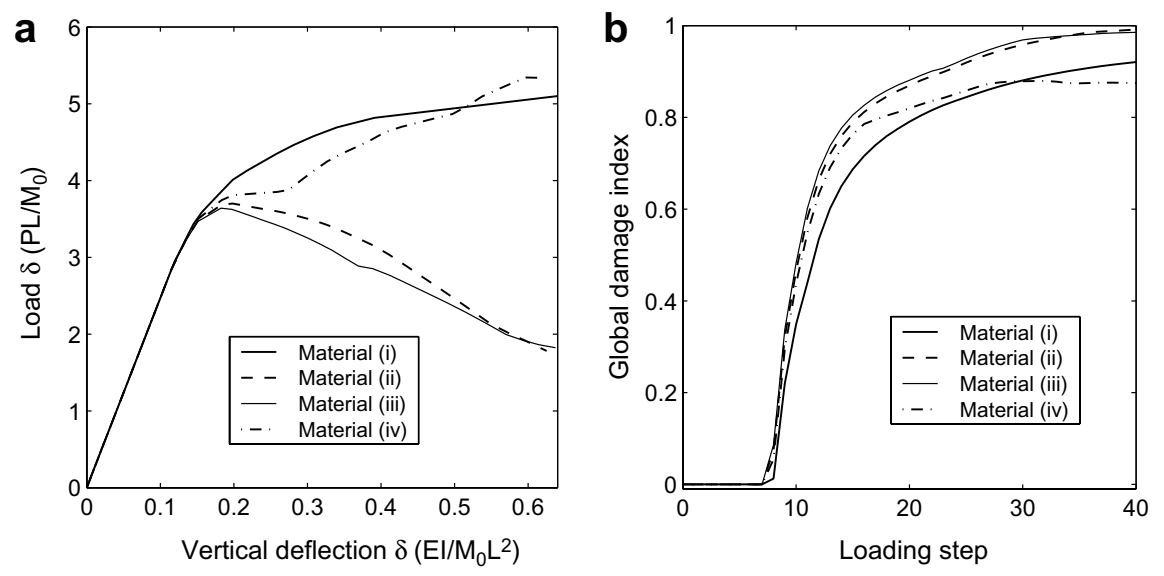

Fig. 19. (a) Load deflection curves. (b) Global damage indices.

Fig. 19a shows the load deflection curve of the point $A$ for the four considered materials. It is possible to appreciate that in the case of the material (iii), after the damaging phase of the composite has been degraded, the response of the structure is purely plastic. In the case of the material (iv), in the large displacements range of the response, the elastic phase dominates the mechanical behavior. Fig. 19b shows the evolution of the global damage index for the four materials. It is worth to note that the damage index corresponding to material (iii) grows faster than the others, but, when the plastic phase dominates the the response (approx. loading step 32), the highest damage index is associated to the material (ii). In the large displacements range, the smallest values of global damage index corresponds to the material (iv) due to the effect of the elastic component.

\section{Conclusions}

The geometrically exact formulation due to Reissner and Simo for 3D beams is considered in the context of initially curved beams and extended to include arbitrary distribution of composite materials in the cross sections. The resulting formulation is used for studying the constitutive and geometric nonlinear behavior of framed structures in the static case.

Constitutive laws for the simple materials are based on thermodynamically consistent formulations allowing to obtain more realistic estimations of the energy dissipation in the nonlinear range. The simple mixing rule for composites is used for modeling complex material behaviors at material point level.

A detailed cross sectional analysis, consistent with the kinematic hypothesis is presented. The proposed method, even when inexact from the point of view of the elasticity theory, gives a computationally convenient way of approximating the strain-stress distribution in the section. Warping variables or iterative procedures for obtaining corrected strain fields are avoided in the present formulation. Consistent tangential constitutive tensors are considered to obtain the tangential beam stiffness matrix by means of cross sectional integration.

A mesh independent response is obtained by means of the regularization of the energy dissipated at constitutive level considering the characteristic length of the volume associated to a specific integration point and the fracture energy of the materials.

Local and global damage indices have been developed based on the ratio between the elastic and nonlinear stresses. The proposed damage indices allow to identify the global load carrying capacity of the structure and the damage state of the different members.

Several numerical examples have been included for the validation of the proposed formulation. The examples include elastic and inelastic response of framed structures with initially straight and curved beams. The verification of the obtention of a mesh independent response for structures presenting softening behavior is carried out. Comparisons with existing literature is performed for the case of plasticity and new results are presented for degrading and composite materials.

\section{Acknowledgements}

This research was partially supported by CEE-FP6 project FP6-505448 (GOCE), Risk Mitigation for Earthquakes and Landslides (LESSLOSS); by the Spanish Government: Ministerio de Fomento and Ministerio de Ciencia y Tecnología. Project Ref. MAT-2003-09768-C03-02, Delamination of reinforced matrix composites (DECOMAR); Ministerio de Educación y Ciencia. Project BIA200308700-C03-02, Numerical simulation of the seismic behavior of structures with energy dissipation devices. Project BIA2005-06952, Study of composite materials for design, reinforcement and retrofit of civil engineering structures (RECOMP). The International Center for Numerical Methods in Enginnering (CIMNE) has also provided the support for this research. All this support is gratefully acknowledged. 


\section{References}

[1] S.S. Antman, Nonlinear Problems of Elasticity, Springer-Verlag, 1991.

[2] F. Armero, D. Ehrlich, Numerical modeling of softening hinges in the Euler-Bernoulli beams, Comput. Struct. 84 (2005) 641-656.

[3] F. Armero, I. Romero, On the objective and conserving integration of geometrically exact rod models, Trends in Computational Structural Mechanics, Lake Constance, Austria, May 2001.

[4] F. Armero, I. Romero, Energy-dissipating momentum-conserving time-stepping algorithms for the dynamic of nonlinear Cosserat rods, Comput. Mech. 31 (2003) 3-26.

[5] J. Argyris, An excursion into large rotations, Comput. Methods Appl. Mech. Engrg. 32 (1982) 85-155.

[6] A.H. Barbat, S. Oller, A. Hanganu, E. Oñate, Viscous damage model for Timoshenko beam structures, Int. J. Solids Struct. 34 (1997) 39533976.

[7] P. Betsch, P. Steinmann, Frame-indifferent beam finite element based upon the geometrically exact beam theory, Int. J. Numer. Methods Engrg. 54 (2002) 1775-1788.

[8] G.E. Blandford, Large deformation analysis of inelastic space truss structures, J. Struct. Engg. 122 (1996) 407-415.

[9] A. Cardona, A. Huespe, Evaluation of simple bifurcation points and post-critical path in large finite rotations problems, Comput. Methods Appl. Mech. Engrg. 175 (1999) 137-156.

[10] A. Cardona, M. Gerardin, A beam finite element non-linear theory with finite rotations, Int. J. Numer. Methods Engrg. 26 (1988) 24032438.

[11] E. Car, S. Oller, E. Oñate, An anisotropic elasto plastic constitutive model for large strain analysis of fiber reinforced composite materials, Comput. Methods Appl. Mech. Engrg. 185 (2000) 245-277.

[12] E. Cosenza, G. Manfredi, Damage indices and damage measures, Prog. Struct. Engrg. Mater. 2 (2000) 50-59.

[13] M.A. Crisfield, Non-Linear Finite Element Analysis of Solids and Structures, vols. 1 \& 2, John Wiley \& Sons, 1998.

[14] M.A. Crisfield, G. Jelenic̀, Objectivity of strain measures in the geometrically exact three-dimensional beam theory and its finiteelement implementation, Royal Soc. 455 (1999) 1125-1147.

[15] L. Davenne, F. Ragueneau, J. Mazars, A. Ibrahimbegović, Efficient approaches to finite element analysis in earthquake engineering, Comput. Struct. 81 (2003) 1223-1239.

[16] M.A. Dides, J.C. De La Llera, A comparative study of concentrated plasticity models in dynamic analysis of building structures, Earthquake Engrg. Struct. Dynam. 34 (2005) 1005-1026.

[17] L. Driemeier, S.P. Baroncini, M. Alves, A contribution to the numerical non-linear analysis of three-dimensional truss system considering large strains, damage and plasticity, Commun. Nonlinear Sci. Numer. Simul. 10 (2005) 515-535.

[18] A.S. Elnashai, A.M. Mwafy, Overstrength and force reduction factors of multistorey reinforced concrete buildings, Struct. Des. Tall Build. 11 (2002) 329-351.

[19] C.A. Felippa, L.A. Crivelli, B. Haugen, A survey of the corecongruential formulation for geometrically nonlinear TL finite elements, Arch. Comput. Methods Engrg. 1 (1994) 1-48.

[20] F. Gruttmann, R. Sauer, W. Wagner, Theory and numerics of threedimensional beams with elastoplastic material behavior, Int. J. Numer. Methods Engrg. 48 (2000) 1675-1702.

[21] A.D. Hanganu, E. Oñate, A.H. Barbat, Finite element methodology for local/global damage evaluation in civil engineering structures, Comput. Struct. 80 (2002) 1667-1687.

[22] HG. Kwak, SP. Kim, Nonlinear analysis of RC beam subject to cyclic loading, J. Struct. Engrg. 127 (2001) 1436-1444.

[23] M. Iura, S.N. Atluri, On a consistent theory, and variational formulation of finitely stretched and rotated 3-D space-curved beams, Computat. Mech. 4 (1989) 73-88.

[24] A. Ibrahimbegović, On finite element implementation of geometrically nonlinear Reissners̀ beam theory: three-dimensional curved beam elements, Comput. Methods Appl. Mech. Engrg. 122 (1995) 1126.

[25] A. Ibrahimbegović, M. Al Mikdad, Quadratically convergent direct calculation of critical points for 3D structures undergoing finite rotations, Comput. Methods Appl. Mech. Engrg. 189 (2000) 107120.

[26] A. Ibrahimbegović, On the choice of finite rotation parameters, Comput. Methods Appl. Mech. Engrg. 149 (1997) 49-71.

[27] A. Ibrahimbegović, R.L. Taylor, On the role of frame-invariance in structural mechanics models at finite rotations, Comput. Methods Appl. Mech. Engrg. 191 (2002) 5159-5176.

[28] A. Ibrahimbegović, H. Shakourzadeh, J.L. Batoz, M. Al Mikdad, Y.Q. Guo, On the role of geometrically exact and second-order theories in buckling and post-buckling analysis of three-dimensional beam structures, Comput. Struct. 61 (1996) 1101-1114.

[29] G. Jelenic̀, M.A. Crisfield, Geometrically exact 3D beam theory: implementation of a strain-invariant finite element for static and dynamics, Comput. Methods Appl. Mech. Engrg. 171 (1999) 141171.

[30] G. Jelenic̀, M. Saje, A kinematically exact space finite strain beam model-finite element formulation by generalized virtual work principle, Comput. Methods Appl. Mech. Engrg. 120 (1995) 131-161.

[31] R.K. Kapania, J. Li, On a geometrically exact curved/twisted beam theory under rigid cross-section assumption, Comput. Mech. 30 (2003) 428-443.

[32] A.J. Kappos, Seismic damage indices for RC buildings: evaluation of concepts and procedures, Prog. Struct. Engrg. Mater. 1 (1997) 78-87.

[33] J.K. Kim, T.G. Lee, Failure behavior of reinforced concrete frames by the combined layered and nonlayered method, Comput. Struct. 48 (1993) 819-825.

[34] J. Lubliner, Thermomechanics of deformable bodies, Department of Civil Engineering University of California, Berkeley, 1985.

[35] J. Lubliner, J. Oliver, S. Oller, Oñate, A plastic-damage model for concrete, Int. J. Solids Struct. 25 (1989) 299-326.

[36] J. Lubliner, On the thermodynamic foundations of non-linear solid mechanics, Int. J. Non-Linear Mech. 7 (1972) 237-254.

[37] B.M. Luccioni, V.C. Rougier, A plastic damage approach for confined concrete, Comput. Struct. 83 (2005) 2238-2256.

[38] J. Mäkinen, H. Marjamäki, Total Lagrangian parametrization of rotation manifold, in: Fifth EUROMECH Nonlinear Dynamics Conference, Eindhoven, Netherlands, 2005, pp. 522-530.

[39] L.E. Malvern, Introduction to the mechanics of a continuous medium, Department of Civil Engineering Englewood Cliffs, NJ, 1969.

[40] A. Pérez Morán, Formulaciones Tangente y Secante en Análisis no Lineal de Vigas de Cosserat, Ph.D. Thesis, Universitat Politècnica de Catalunya, 2005.

[41] A. Neuenhofer, F.C. Filippou, Evaluation of the nonlinear frame finite-element models, J. Struct. Engrg. 123 (1997) 958-966.

[42] J. Oliver, M. Cervera, S. Oller, J. Lubliner, Isotropic damage models and smeared crack analysis of concrete, Proceedings 2nd ICCAADCS, Zell Am See, Austria, vol. 2, Pineridge Press, 1990, pp. 945958.

[43] R.W. Ogden, Non-linear Elastic Deformations, Dover Publications, Inc., 1997.

[44] S. Oller, A.H. Barbat, Moment-curvature damage model for bridges subjected to seismic loads, Comput. Methods Appl. Mech. Engrg. 195 (2006) 4490-4511.

[45] S. Oller, E. Oñate, J. Miquel, S. Botello, A plastic damage constitutive model for composites materials, Int. J. Solids Struct. 33 (1996) 25012518.

[46] S. Oller, E. Oñate, J. Miquel, Mixing anisotropic formulation for the analysis of composites, Commun. Numer. Methods Engrg. 12 (1996) 471-482.

[47] S. Pajunen, Large elasto-plastic analysis of beams using kinematically exact elements, Commun. Numer. Methods Engrg. 16 (2000) 497504. 
[48] I. Papaioannou, M. Fragiadakis, M. Papadrakakis, Inelastic analysis of framed structures using the fiber approach, in: Proceedings of the 5th International Congress on Computational Mechanics (GRACM 05), Limassol, Cyprus, 2005, pp. 231-238.

[49] M.S. Park, B.C. Lee, Geometrically non-linear and elastoplastic threedimensional shear flexible beam element of Von-Mises-type hardening material, Int. J. Numer. Methods Engrg. 39 (1996) 383-408.

[50] P.K.V.V. Nukala, D.W. White, A mixed finite element for threedimensional nonlinear analysis of steel frames, Comput. Methods Appl. Mech. Engrg. 193 (2004) 2507-2545.

[51] E. Reissner, On one-dimensional large-displacement finite-strain beam theory, Stud. Appl. Math. 11 (1973) 87-95.

[52] E. Reissner, On one-dimensional finite-strain beam theory: the plane problem, J. Appl. Math. Phys. 23 (1972) 795-804.

[53] I. Romero, F. Armero, An objective finite element formulation of the kinematics of geometrically exact rods and its use in the formulation of an energy-momentum conserving scheme in dynamics, Int. J. Numer. Methods Engrg. 54 (2002) 1683-1716.

[54] M. Saje, G. Turk, A. Kalagasidu, B. Vratanar, A kinematically exact finite element formulation of elastic-plastic curved beams, Comput. Struct. 67 (1998) 197-214.

[55] M.H. Scott, G.L. Fenves, Plastic hinge integration methods for the force-based beam-column elements, J. Struct. Engrg. 132 (2006) 244 252.

[56] Y. Shao, S. Aval, A. Mirmiran, Fiber-element model for cyclic analysis of concrete-filled fiber reinforced polymer tubes, J. Struct. Engrg. 131 (2005) 292-303.

[57] G. Shi, S.N. Atluri, Elasto-plastic large deformation analysis of space-frames: a plastic hinge and stress-based explicit derivation of tangent stiffness, Int. J. Numer. Methods Engrg. 26 (1988) 589-615.
[58] J.C. Simo, A finite strain beam formulation. The three-dimensional dynamic problem. Part I, Comput. Methods Appl. Mech. Engrg. 49 (1985) 55-70.

[59] J.C. Simo, L. Vu-Quoc, A three-dimensional finite-strain rod model. Part II: Computational aspects, Comput. Methods Appl. Mech. Engrg. 58 (1986) 79-116.

[60] J.C. Simo, L. Vu-Quoc, On the dynamics in space of rods undergoing large motions - a geometrically exact approach, Comput. Methods Appl. Mech. Engrg. 66 (1988) 125-161.

[61] J.C. Simo, The (symmetric) Hessian for geometrically nonlinear models in solid mechanics: intrinsic definition and geometric interpretation, Comput. Methods Appl. Mech. Engrg. 96 (1992) 189-200.

[62] J.C. Simo, L. Vu-Quoc, A geometrically exact rod model incorporating shear and torsion-warping deformation, Int. J. Solids Struct. 27 (1991) 371-393.

[63] J.C. Simo, K.D. Hjelmstad, R.L. Taylor, Numerical simulations of elasto-viscoplastic response of beams accounting for the effect of shear, Comput. Methods Appl. Mech. Engrg. 42 (1984) 301-330.

[64] M. Shugyo, Elastoplastic large deflection analysis of three-dimensional steel frames, Journal of Structural Engineering 129 (2003) 1259-1267.

[65] G. Turkalj, J. Brnic, J. Prpic-Orsic, ESA formulation for large displacement analysis of framed structures with elastic-plasticity, Comput. Struct. 82 (2004) 2001-2013.

[66] A.M. Yu, X.G. Yang, G.H. Nie, Generalized coordinate for warping of naturally curved and twisted beams with general cross-sectional shapes, Int. J. Solids Struct. 43 (2006) 2853-2867.

[67] D. Zupan, M. Saje, Analytical integration of the stress field and tangent material moduli over concrete cross-sections, Comput. Struct. 83 (2005) 2368-2380. 